\title{
Reconstruction of top-quark mass effects in Higgs pair production and other gluon-fusion processes
}

\author{
Ramona Gröber, Andreas Maier and Thomas Rauh \\ Institute for Particle Physics Phenomenology, University of Durham, \\ South Rd, Durham, United Kingdom \\ E-mail: ramona.groeber@durham.ac.uk, andreas.maier@durham.ac.uk, \\ thomas.j.rauh@durham.ac.uk
}

ABSTRACT: We propose a novel method for the treatment of top-quark mass effects in the production of $H^{(*)}, H H, H Z$ and $Z Z$ final states in gluon fusion. We show that it is possible to reconstruct the full top-quark mass dependence of the virtual amplitudes from the corresponding large- $m_{t}$ expansion and the non-analytic part of the amplitude near the top-quark threshold $\hat{s}=4 m_{t}^{2}$ with a Padé ansatz. The reliability of our method is clearly demonstrated by a comparison with the recent NLO result for Higgs pair production with full top-quark mass dependence.

KeYwords: Effective Field Theories, Higgs Physics, Perturbative QCD

ARXIV EPRINT: 1709.07799 


\section{Contents}

1 Introduction 1

2 The method 3

2.1 Padé approximation for $g g \rightarrow H^{(*)} 4$

2.2 Padé approximation for $g g \rightarrow H H \quad 9$

3 The amplitude near threshold $\quad 10$

$\begin{array}{lll}3.1 & \text { Structure of the amplitude near threshold } & 10\end{array}$

$\begin{array}{ll}3.2 & \text { Computation of the non-analytic terms } \\ & 12\end{array}$

4 Comparison with the exact result $\quad 16$

$\begin{array}{ll}4.1 \text { Numerical setup } & 16\end{array}$

$\begin{array}{ll}4.2 \text { Comparison at LO } & 16\end{array}$

$\begin{array}{lll}4.3 & \text { Comparison at NLO } & 17\end{array}$

5 Conclusions and outlook $\quad 20$

$\begin{array}{lr}\text { A Subtractions } & 22\end{array}$

B Expansion of the P-wave Green function 22

C Results for the $g g \rightarrow H H$ form factors near threshold 23

\section{Introduction}

Gluons are ubiquitous at the LHC, and gluon fusion is among the phenomenologically most interesting production mechanisms. Specifically, the production of final states including one or more Higgs bosons is typically dominated by gluon fusion, with a virtual top-quark loop mediating the interaction to the Higgs bosons.

Precise predictions for such processes are indispensable for measuring the properties of the Higgs boson. On the one hand, gluon fusion processes experience large $K$-factors. ${ }^{1}$ Examples include a $K$-factor of 2.3 for single Higgs and 1.7 for Higgs pair production at next-to-leading order (NLO) [4-10] which clearly demonstrates the importance of taking higher-order corrections into account. On the other hand, calculating these higher-order corrections is extremely challenging. Gluon fusion is a loop-induced process, and the topquark mass introduces an additional scale in the loop integrals. While the NLO corrections to single-Higgs production have been known analytically for some time [5-8], the calculation

\footnotetext{
${ }^{1}$ See $[1-3]$ for a discussion of 'timelike' logarithms in gluon fusion and their resummation which reduces the size of perturbative corrections significantly.
} 
of NLO corrections to processes with more than one final-state particle is still subject of on-going work. For di-Higgs production, which requires the evaluation of two-loop integrals with four scales, numerical results have only become available recently [9, 10].

To make higher-order computations feasible an effective field theory (EFT), where the top quark has been integrated out in the limit of an infinite top-quark mass, $m_{t} \rightarrow \infty$, has been used extensively in the literature. In this approximation, results are available at NNNLO for single Higgs production [4, 11] and at NNLO for Higgs pair production [12, 13], and for other gluon fusion processes, i.e. $g g \rightarrow Z Z, g g \rightarrow H j$ at NNLO [14-17] and $g g \rightarrow H Z$. Beyond the infinite top mass limit, several results have also been obtained in the large- $m_{t}$ expansion (LME) for a number of processes listed here:

- $g g \rightarrow H:$ up to $1 / m_{t}^{6}$ at NNLO [18-22], including $g g \rightarrow H g$ at NLO

- $g g \rightarrow H H$ : up to $1 / m_{t}^{12}$ in [23] and $1 / m_{t}^{8}$ in [24] at NLO; up to $1 / m_{t}^{4}$ at NNLO [23]

- $g g \rightarrow H Z:$ up to $1 / m_{t}^{8}[25]$ at NLO

- $g g \rightarrow Z Z$ : up to $1 / m_{t}^{12}$ in [26] and $1 / m_{t}^{8}$ in [27] at NLO

The expansions can be rescaled with the exact leading order (LO) result

$$
\mathrm{d} \sigma_{\mathrm{NLO}}^{\text {rescaled LME }} / \mathrm{d} X=\frac{\mathrm{d} \sigma_{\mathrm{NLO}}^{\mathrm{LME}} / \mathrm{d} X}{\mathrm{~d} \sigma_{\mathrm{LO}}^{\mathrm{LME}} / \mathrm{d} X} \mathrm{~d} \sigma_{\mathrm{LO}}^{\text {exact }} / \mathrm{d} X
$$

where $\mathrm{d} \sigma / \mathrm{d} X$ indicates the differential cross section with respect to some quantity $X$. For inclusive Higgs production this yields good agreement with the exact NLO result [5-8]. The comparison with the exact Higgs pair production result has however revealed the shortcomings of the approximation (1.1) for this process $[9,10]$. This issue is especially pronounced when distributions are considered.

Here, we advocate a different approach, based on conformal mapping and the construction of Padé approximations from expansions in different kinematical regimes of the amplitude. This strategy has first been introduced for heavy-quark current correlators $\Pi^{(j)}\left(q^{2} /\left(4 m_{q}^{2}\right)\right)[28,29]$ and applied successfully up to four-loop order [30-32]. The approximation can be improved systematically by including more information from the various kinematic limits. In fact, the three-loop approximation is indistinguishable from the results of an exact numeric computation [33]. In [28], it has also been shown for the decay $H \rightarrow \gamma \gamma$ that a Padé reconstruction of the top mass effects from the asymptotic expansion in a large top mass yields excellent agreement with the full NLO decay rate. Like for heavy-quark correlators and the $H \rightarrow \gamma \gamma$ decay rate, the amplitude for Higgs production in gluon fusion only depends on one ratio of scales and the application of the method is straightforward. However, the amplitudes for the remaining processes listed above depend on 4-5 scales. Padé approximations based on the LME terms alone have been used to reconstruct the interference contribution in $g g \rightarrow Z Z[26]$. An attempt to reconstruct the $g g \rightarrow H Z$ cross 
section has been made in [25]. ${ }^{2}$ In this work, we show how such an approximation can be improved drastically by also taking into account expansions in other kinematic regions, using Higgs pair production as an example.

Measuring di-Higgs production at the LHC allows to directly determine the trilinear Higgs boson self-coupling $\lambda_{3}$ [34-36], which serves as a probe of the shape of the Higgs potential and is a crucial test of the mechanism of electroweak symmetry breaking in nature. While the couplings of the Higgs boson to the gauge bosons and third-generation fermions have been firmly established to be Standard Model like within 10-20\% [37-39], constraining the trilinear self-coupling is highly challenging. With $3000 \mathrm{fb}^{-1}$ of data the estimated bounds are $0.2<\lambda_{3} / \lambda_{3}^{S M}<7.0$ (neglecting systematic uncertainties) [40]. Current bounds from Higgs pair production final states limit the trilinear Higgs self-coupling between $-8.8<\lambda_{3} / \lambda_{3}^{S M}<15.0$ [41]. Under the assumption that only the trilinear Higgs self-coupling is modified, bounds can be obtained from single Higgs production through the electroweak corrections [42-45] or from electroweak precision observables [46, 47]. However, the current bounds are still above the limits from perturbativity [48].

Precise theory predictions are crucial in the extraction of $\lambda_{3}$ from the cross section measurements. It is evident already at leading order (LO) that the LME alone is not sufficient. In fact, as shown in figure 1, the cross section is dominated by energies of about $400 \mathrm{GeV}$, whereas the LME breaks down at the top pair-production threshold around $2 m_{t} \approx 350 \mathrm{GeV}$. As we will show, constructing Padé approximations from the LME can ameliorate this problem to some degree, but not solve it completely. The reason for this is that, above the top threshold, the production amplitude receives non-analytic contributions, which cannot be reproduced by the purely rational Padé approximants. Incorporating these nonanalytic threshold corrections enhances the quality of the approximation dramatically in the dominant kinematic region and thus leads to a much improved prediction for the total cross section.

The outline of this paper is as follows: in section 2 we introduce our method for single Higgs production and then show how it can be generalized to the case of Higgs pair production. The computation of the additional input terms from the expansion around the top threshold is described in section 3. In section 4 we perform a detailed comparison of both the LO and NLO Padé approximation with the full LO result and the recent NLO results $[9,10]$, respectively. We conclude in section 5 and offer an outlook over possible applications of our method.

\section{The method}

We first discuss the construction of a Padé approximation for the simple case of the virtual amplitude $\mathcal{A}_{g g \rightarrow H^{(*)}}$ in section 2.1 and then generalize the approach to Higgs pair production in section 2.2 .

\footnotetext{
${ }^{2}$ The method presented below depends crucially on the analytic structure of the amplitude, whereas [25] considers Padé approximants to the differential cross section, which is not an analytic function of the ratio $\hat{s} /\left(4 m_{t}^{2}\right)$ near $m_{t} \rightarrow \infty$. Therefore, the approach used in [25] does not yield an adequate description above the top threshold and the improvement from employing a conformal mapping is marginal.
} 


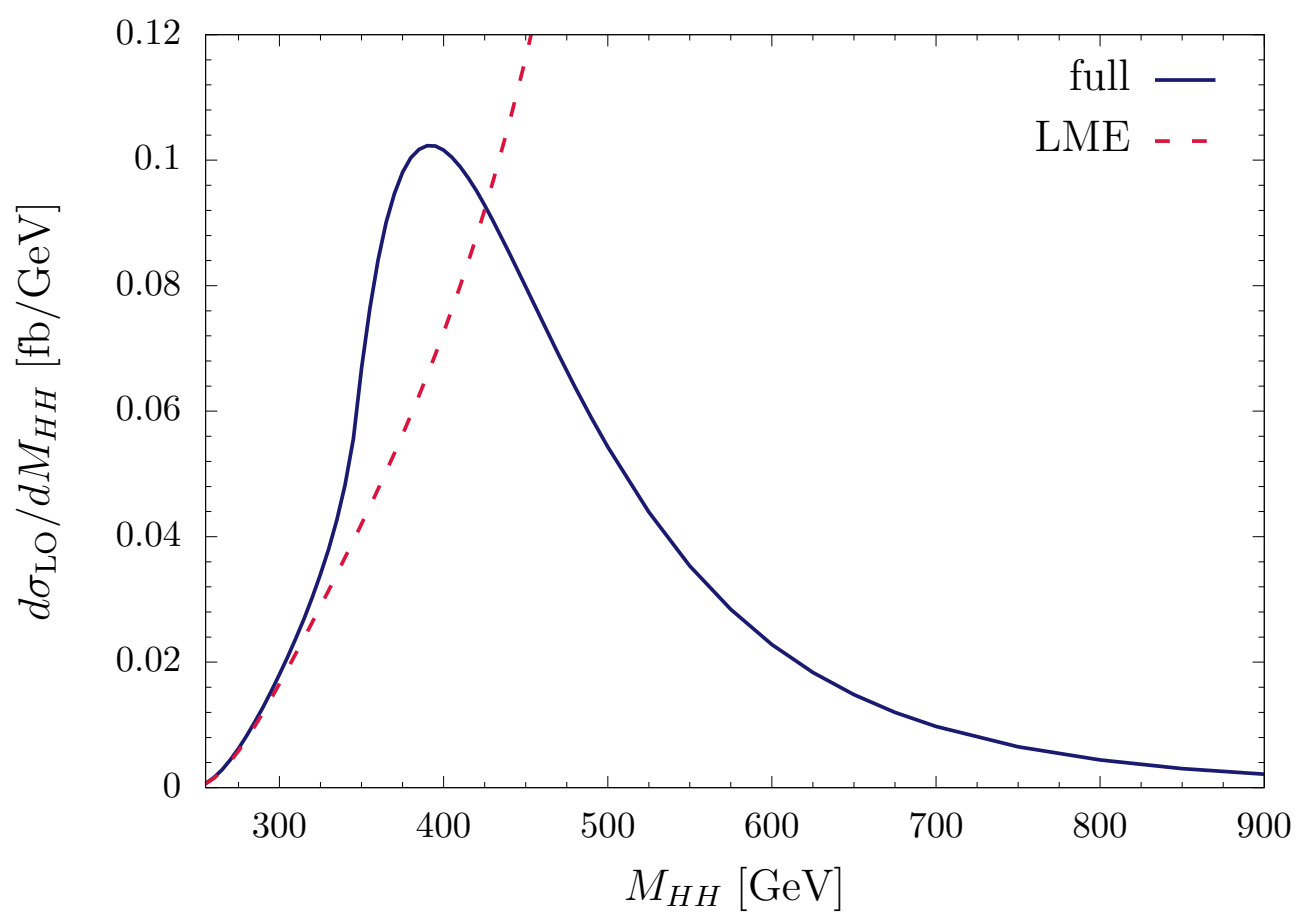

Figure 1. Invariant Higgs mass distribution for the full LO cross section (dark blue) and the large mass expansion (LME) up to $\mathcal{O}\left(1 / m_{t}^{8}\right)$ as given in ref. [24] (red-dashed).

\subsection{Padé approximation for $g g \rightarrow H^{(*)}$}

The LO diagram for the production of an off-shell Higgs in gluon fusion is shown in figure 2 (left). The corresponding amplitude can be expressed through a dimensionless form factor $F_{\triangle}$ that only depends on the variable $z=(\hat{s}+i 0) /\left(4 m_{t}^{2}\right)$

$$
\mathcal{A}_{a b}^{\mu \nu}\left(g\left(p_{1}, \mu, a\right), g\left(p_{2}, \nu, b\right) \rightarrow H^{(*)}\left(p_{H}\right)\right)=\frac{y_{t} \hat{s}}{\sqrt{2} m_{t}} \frac{\alpha_{s}}{2 \pi} \delta_{a b} T_{F} A_{1}^{\mu \nu} F_{\triangle}(z)
$$

where $\hat{s}=\left(p_{1}+p_{2}\right)^{2}=p_{H}^{2}, y_{t}=\sqrt{2} m_{t} / v$ is the top Yukawa coupling, $T_{F}=1 / 2$ and

$$
A_{1}^{\mu \nu}=g^{\mu \nu}-\frac{p_{1}^{\nu} p_{2}^{\mu}}{p_{1} \cdot p_{2}} .
$$

The form factor $F_{\triangle}$ is normalized such that

$$
F_{\triangle} \stackrel{m_{t} \rightarrow \infty}{\longrightarrow} \frac{4}{3}+\mathcal{O}\left(\alpha_{s}\right)
$$

The leading-order contribution to the form factor is analytic in the entire complex plane with the exception of a branch cut for real $z \geq 1$ due to on-shell $t \bar{t}$ cuts. At NLO, massless cuts like the one shown in the right of figure 2 introduce a branch cut starting at $z=0$. However, the branch cut can be made explicit

$$
\begin{aligned}
F_{\triangle} & =F_{\triangle}^{1 l}+\frac{\alpha_{s}}{\pi} F_{\triangle}^{2 l}+\mathcal{O}\left(\alpha_{s}^{2}\right) \\
& =F_{\triangle}^{1 l}+\frac{\alpha_{s}}{\pi}\left[C_{F} F_{\triangle, C_{F}}^{2 l}+C_{A}\left(F_{\triangle, C_{A}}^{2 l}+F_{\triangle, C_{A}, \ln }^{2 l} \ln (-4 z)\right)\right]+\mathcal{O}\left(\alpha_{s}^{2}\right),
\end{aligned}
$$



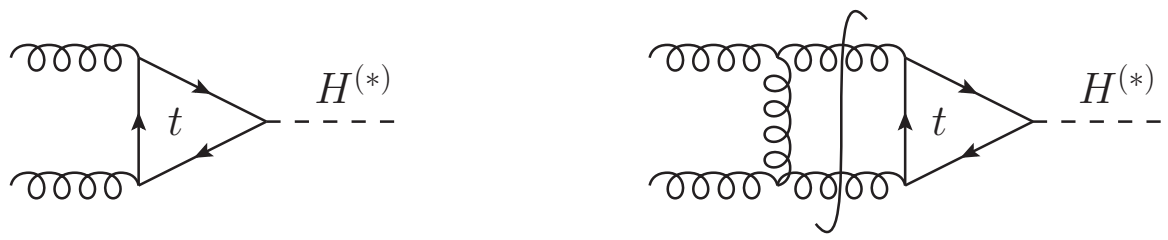

Figure 2. The LO diagram for Higgs production in gluon fusion (left) and an example for a NLO diagram that contains a branch cut starting at $\hat{s}=0$ (right).

such that all the $F_{\triangle, x}^{i l}$ (with $i=1,2$ and $x=C_{F}, C_{A},\left(C_{A}, \ln \right)$ ) on the right-hand side are again analytic except for real $z \geq 1$. In $F_{\triangle, C_{A}}^{2 l}$, IR divergences in the amplitude have been subtracted as described in ref. [24]. We can now apply the conformal transformation [28]

$$
z=\frac{4 \omega}{(1+\omega)^{2}}
$$

to map the entire complex $z$ plane onto the unit disc $|\omega| \leq 1$ while the branch cut at $z \geq 1$ is mapped onto the perimeter. The physical branch $\operatorname{Im}(z)>0$ corresponds to the upper semicircle, starting at $\omega(z=1)=1$ and ending at $\omega(z \rightarrow \infty+i 0)=-1$. With this mapping, the $F_{\triangle, x}^{i l}$ are analytic functions of $\omega$ inside the unit circle. We approximate them using a Padé ansatz

$$
[n / m](\omega)=\frac{\sum_{i=0}^{n} a_{i} \omega^{i}}{1+\sum_{j=1}^{m} b_{j} \omega^{j}}
$$

with a total of $n+m+1$ coefficients. They can be fixed by imposing conditions stemming from known expansions of the approximated function. In many cases it is found that diagonal Padé approximants with $n=m$ provide the best description. Indeed, we find that this also holds for our analysis. We therefore discard approximants that are too far away from the diagonal, as detailed below.

The LME for the form factor $F_{\triangle}$ has been given up to terms of the order $z^{4}$ in [8]. The conformal mapping (2.5) transforms this into constraints on the derivatives of the Padé approximant at $\omega=0$. Furthermore the form factor vanishes for $z \rightarrow \infty$ as $F_{\triangle}(z)=\mathcal{O}(1 / z)$ since $\hat{s} \sim z$ has been factored out in (2.1). In a direct approach this would imply the constraint $[n / m](\omega=-1)=0$. Instead, we construct the Padé approximant for the rescaled form factor

$$
[n / m](\omega) \simeq\left[1+a_{R} z(\omega)\right] F_{\triangle}(z(\omega)),
$$

where $a_{R}$ is a free parameter. This serves a double purpose. First, it removes the spurious constraint at $\omega=-1$ which implies that the dimensionality of the non-linear system of equations that determines the coefficients of the Padé approximant is reduced by one. Secondly, the variation of the parameter $a_{R}$ allows us to test the stability of the ansatz and to assign an uncertainty to the reconstruction.

A set of Padé approximants with $n+m=4$ can be constructed based only on the constraints from the LME up to $\mathcal{O}\left(z^{4}\right)$. The Padé ansatz (2.6) has $m$ poles in the $\omega$ plane. 
Here, and in the remainder of this work, we eliminate a subset of Padé approximants based on the positions of these poles. Since the amplitude is analytic inside the unit disc, the canonical selection criterion is to exclude approximants with poles at $|\omega| \leq 1+\delta$, where $\delta>0$ should be chosen such that no unphysical resonances, caused by nearby poles, are observed in the amplitude. We find, however, that this criterion proves too restrictive as it excludes almost all approximants. Thus, we relax the selection criterion and exclude approximants with poles in the region corresponding to values of $z$ with $0 \leq \operatorname{Re}(z) \leq 8$ and $-1 \leq \operatorname{Im}(z) \leq 1$, thereby excluding poles in the vicinity of the phenomenologically relevant region $0.13 \lesssim z \lesssim 5$. We have checked the stability of the results under variation of the exclusion region. The result is shown in figure 3 and compared to the exact expression for the form factor [5-8]. At LO the agreement is good, whereas at NLO the Padé curves become unstable under variations of $a_{R}$ and $n / m$ and show significant deviations from the exact result for energies near and above the top threshold $z \gtrsim 1$.

We can gain some insight into this deviation by studying the expansion of the form factor around the top threshold. In particular we are interested in the non-analytic terms in the expansion in $(1-z)$ which can be determined with the help of a factorization formula as discussed below in section 3 . Our results take the form

$$
\begin{aligned}
& F_{\triangle}^{1 l} \stackrel{z \rightarrow 1}{\asymp} 2 \pi(1-z)^{3 / 2}+\frac{13 \pi}{3}(1-z)^{5 / 2}+\mathcal{O}\left((1-z)^{7 / 2}\right), \\
& F_{\triangle, C_{F}}^{2 l} \stackrel{z \rightarrow 1}{\asymp} \pi^{2}(1-z) \ln (1-z)-\frac{\pi\left(40-3 \pi^{2}\right)}{12}(1-z)^{3 / 2}+\frac{2 \pi^{2}}{3}(1-z)^{2} \ln (1-z) \\
& +\mathcal{O}\left((1-z)^{5 / 2}\right) \\
& F_{\triangle, C_{A}}^{2 l} \stackrel{z \rightarrow 1}{\overbrace{}^{1}}-\frac{\pi\left(3 \pi^{2}-4\right)}{12}(1-z)^{3 / 2}+\mathcal{O}\left((1-z)^{5 / 2}\right), \\
& F_{\triangle, C_{A}, \ln }^{2 l} \stackrel{z \rightarrow 1}{\leftrightarrows} \mathcal{O}\left((1-z)^{5 / 2}\right),
\end{aligned}
$$

where we have used the symbol $\asymp$ to denote that terms that are analytic in $(1-z)$ have been dropped on the right-hand side. We observe that threshold logarithms $\ln (1-z)$, which cannot be reproduced by the Padé ansatz, appear at NLO. Having determined the coefficients of the logarithmic terms at the first two orders we can however subtract them from the form factor and apply the Padé approximation to the subtracted function. Taking a function $f(z)$ with the threshold expansion

$$
f(z) \stackrel{z \rightarrow 1}{\overbrace{}^{1}} c_{1} \sqrt{1-z}+c_{2}(1-z) \ln (1-z)+c_{3}(1-z)^{3 / 2}+c_{4}(1-z)^{2} \ln (1-z)+\ldots
$$

as an example we define

$$
\tilde{f}(z)=f(z)-c_{2} s_{2}(z)-\left(c_{4}-\frac{c_{2}}{3}\right) s_{4}(z),
$$

where $s_{2,4}$ are constructed such that their leading non-analytic terms in the threshold expansion are given by $(1-z) \ln (1-z)$ and $(1-z)^{2} \ln (1-z)$, respectively. In addition, the subtraction terms must be analytic around $z=0$ and at most logarithmically divergent for $z \rightarrow \infty .^{3}$ Apart from these constraints, the exact form of the subtraction functions is

\footnotetext{
${ }^{3}$ In principle, non-logarithmic poles of the form $z^{n}$ are also allowed, but these have to cancel against corresponding poles in the Padé approximation.
} 

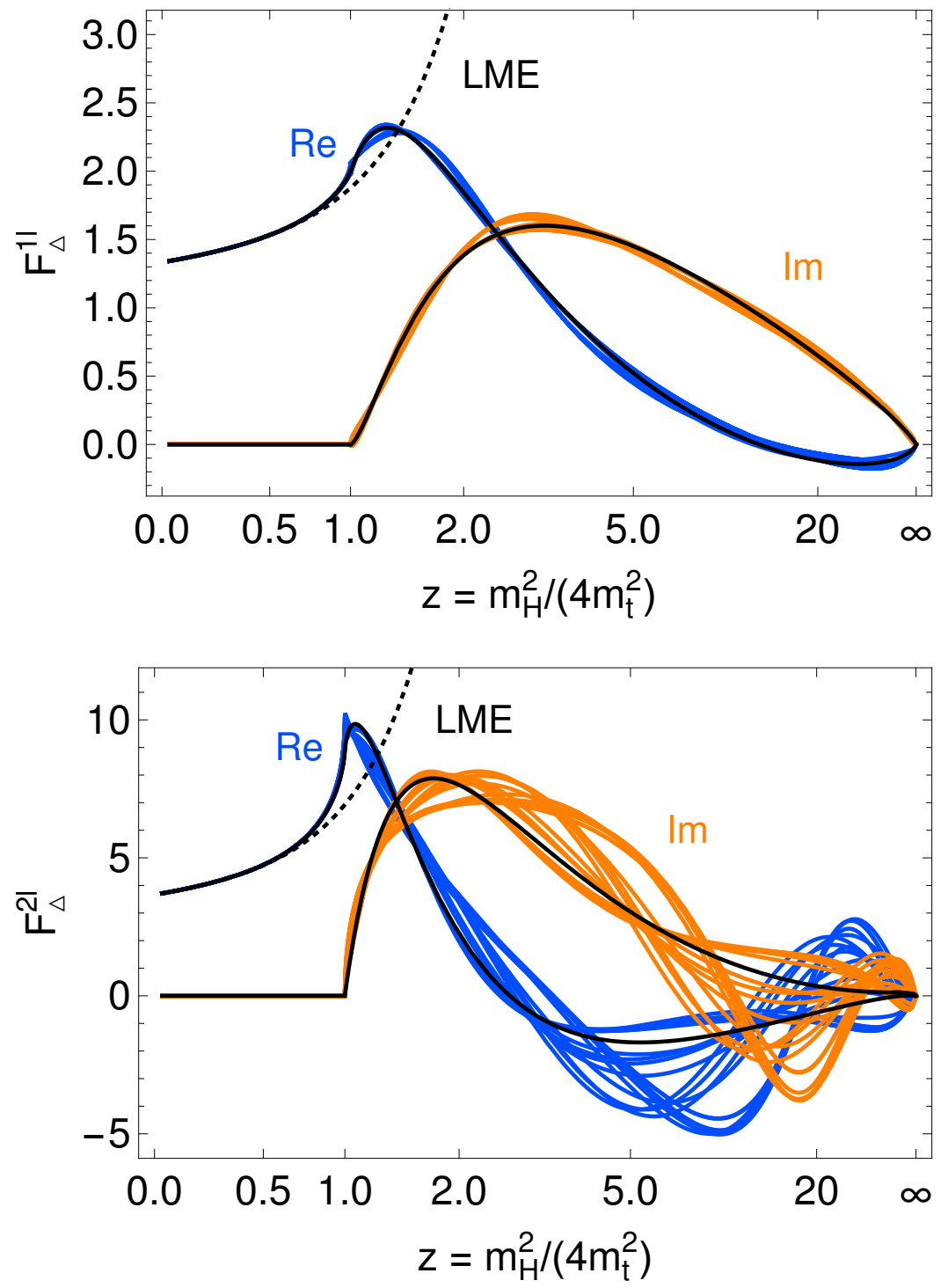

Figure 3. Padé approximants for $F_{\triangle}$ at LO (top) and NLO (bottom) constructed using only the LME up to the order $1 / m_{t}^{8}$ as input. Shown are the real/imaginary part of the Padé approximants (blue/orange) and the exact results (black). We constructed in total 20 approximants of the types $[1 / 3],[2 / 2]$ and [3/1] for random values of $a_{R}$ in the range [0.1,10], while approximants with poles in the rectangle $\operatorname{Re}(z) \in[0,8]$ and $\operatorname{Im}(z) \in[-1,1]$ have been excluded since they can cause unphysical resonances in the form factor.

arbitrary. Our choice for the functions $s_{2,4}$ can be found in appendix A. The threshold expansion of $\tilde{f}$ is free of logarithms up to and including the order $(1-z)^{2}$. An improved approximation of the original function $f$ is then given by

$$
f(z) \simeq[n / m]_{\tilde{f}}(\omega(z))+c_{2} s_{2}(z)+\left(c_{4}-\frac{c_{2}}{3}\right) s_{4}(z),
$$

where the Padé approximant $[n / m]_{\tilde{f}}$ is constructed from the expansion terms of the subtracted function $\tilde{f}$. 

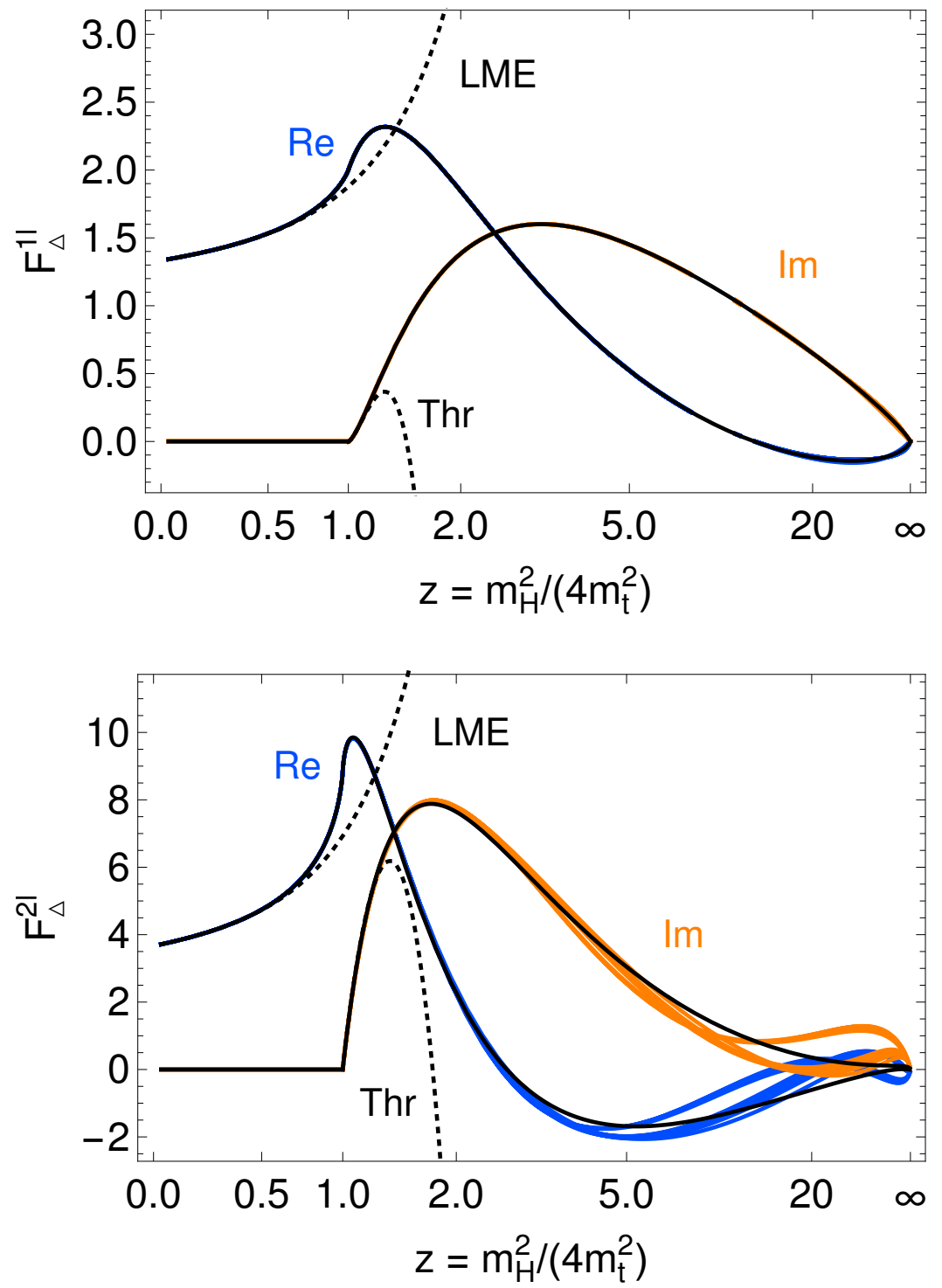

Figure 4. We show the same comparison as in figure 3 but for Padé approximants based on the LME and the threshold expansion. Only [5/2], [4/3], [3/4] and [2/5] approximants were constructed at LO and only [4/2], [3/3] and [2/4] approximants were constructed at NLO.

In addition, the non-integer powers of $(1-z)$ in eqs. (2.8)-(2.11) imply constraints on the derivatives of the Padé approximation at $\omega=1$. By using all the available constraints we can construct approximants with a total of $n+m+1=8$ coefficients at LO and $n+m+1=7$ coefficients at NLO. The results are given in figure 4 and show perfect agreement with the exact LO form factor in the full energy range. At NLO the agreement is excellent up to $z \sim 2.5$ where tiny deviations begin to emerge. For very large $z$, outside the phenomenologically relevant energy range, the approximants have unphysical extrema. We suspect that they could be removed by including information from the small $m_{t}$ expansion (SME) of the form factors in the construction. 
An alternative implementation is obtained by performing additional subtractions for the root terms by employing the functions $s_{1,3,5}$ in appendix A, thereby removing all known non-analytic terms in the expansion. This yields the same number of constraints on the Padé approximant. In the following we will only use the subtraction functions $s_{2,4}$, since we find no significant differences between the two approaches.

\subsection{Padé approximation for $g g \rightarrow H H$}

The amplitude for the process $g g \rightarrow H H$ can be parametrized by two dimensionless form factors $F_{1,2}$

$$
\mathcal{A}_{a b}^{\mu \nu}\left(g\left(p_{1}, \mu, a\right), g\left(p_{2}, \nu, b\right) \rightarrow H\left(p_{3}\right) H\left(p_{4}\right)\right)=y_{t}^{2} \frac{\alpha_{s}}{2 \pi} \delta_{a b} T_{F} z\left[A_{1}^{\mu \nu} F_{1}+A_{2}^{\mu \nu} F_{2}\right],
$$

where $\hat{s}=\left(p_{1}+p_{2}\right)^{2}, \hat{t}=\left(p_{1}-p_{3}\right)^{2}, \hat{u}=\left(p_{1}-p_{4}\right)^{2}, \hat{s}+\hat{t}+\hat{u}=2 m_{H}^{2}, A_{1}^{\mu \nu}$ is given in $(2.2)$ and

$$
A_{2}^{\mu \nu}=g^{\mu \nu}+\frac{p_{3}^{2} p_{1}^{\nu} p_{2}^{\mu}-2\left(p_{3} \cdot p_{2}\right) p_{1}^{\nu} p_{3}^{\mu}-2\left(p_{3} \cdot p_{1}\right) p_{3}^{\nu} p_{2}^{\mu}+2\left(p_{1} \cdot p_{2}\right) p_{3}^{\mu} p_{3}^{\nu}}{p_{T}^{2}\left(p_{1} \cdot p_{2}\right)}
$$

with

$$
p_{T}^{2}=\frac{\hat{t} \hat{u}-m_{H}^{4}}{\hat{s}} .
$$

Given that there are four independent scales the dimensionless form factors depend on three ratios

$$
F_{i}=F_{i}\left(r_{H} \equiv \frac{m_{H}^{2}}{\hat{s}}, r_{p_{T}} \equiv \frac{p_{T}^{2}}{\hat{s}}, z\right), \quad i=1,2 .
$$

This implies that their analytic structure is much more complicated than it was the case for $F_{\triangle}$. For instance, there are branch cuts in the complex $\hat{t}$ and $\hat{u}$ planes above the thresholds $\hat{t} \geq 4 m_{t}^{2}$ and $\hat{u} \geq 4 m_{t}^{2}$. These are, however, not kinematically accessible for external momenta that are both real and on shell. Furthermore, for $z \geq 1 / r_{H} \geq 4$ there is also a discontinuity from cuts corresponding to the processes $g g \rightarrow t \bar{t} H$ and $H \rightarrow t \bar{t}$ which are, however, not accessible for the physical Higgs and top masses. In the limit of small quark masses, $z \rightarrow \infty$, where this type of cut is present, the recent analytical computation of the NLO virtual amplitudes for Higgs plus jet production $[49,50]$ has revealed a rather complicated structure of logarithms in the soft and (in particular) the collinear limit which is presently not fully understood.

Here, we take a practitioners approach and note that when $r_{H}$ and $r_{p_{T}}$ are kept fixed we can separate massless cuts as in (2.4) and again end up with functions that are analytic in $z$ apart from a branch cut for real $z>1$. Therefore it is possible to approximate the top-quark mass dependence of the form factors at a given phase-space point, i.e. for fixed $m_{H}^{2}, \hat{s}$ and $p_{T}^{2}$, by constructing a Padé approximant that describes the dependence on the variable $z$.

We find that the inclusion of the top threshold terms, as described for the triangle form factor (2.4) in section 2.1, is of even greater importance for the construction of Padé approximants for the form factors (2.18) than for $F_{\triangle}$. The computation of these terms is described in the following section 3 and our results are given in appendix C. Readers who 
are mostly interested in the phenomenological aspects may prefer to proceed to section 4 . There, we assess the reliability of our approach for Higgs pair production by performing a detailed comparison with the exact NLO results.

\section{The amplitude near threshold}

In this section the computation of the non-analytic terms in the threshold expansion of the form factors defined in section 2 is described. Factorization formulae for the inclusive production cross section of heavy-particle pairs near threshold have been developed in [51-55] and applied to a number of processes [56-63]. The approach is based on the factorization of forward-scattering amplitudes which are related to the inclusive cross section by the optical theorem. We have extended the factorization formula to the $g g \rightarrow H^{(*)}, H H, H Z, Z Z$ amplitudes. Only the basic aspects are sketched here and the reader is referred to the original literature [51-55] for a detailed derivation and discussion.

\subsection{Structure of the amplitude near threshold}

Near the threshold, $z \rightarrow 1$, the top quarks can only be on shell if they are non-relativistic. This implies a large hierarchy between the top mass $m_{t}$, its typical momentum $m_{t} \sqrt{1-z}$ and its kinetic energy $m_{t}(1-z)$ which set the hard, soft and ultrasoft scale, respectively. Therefore, an effective field theory (EFT) can be constructed by integrating out the hard and soft scale. Then, the only dynamical modes left are non-relativistic top quarks, collinear and ultrasoft gluons and the external fields. The EFT describes the interactions of the remaining modes and is based on potential non-relativistic QCD (PNRQCD) [64-69] and Soft Collinear Effective Theory (SCET) [70-75]. The amplitudes for $g g \rightarrow F$ with final states $F=H^{(*)}, H H, H Z, Z Z$ are given by the master formula (cf. [51, 52])

$$
\begin{aligned}
i \mathcal{A}_{g g \rightarrow F} \stackrel{z \rightarrow 1}{=} \sum_{k, l} C_{g g \rightarrow t \bar{t}}^{(k)} C_{t \bar{t} \rightarrow F}^{(l)} \int d^{4} x\left\langle F\left|T\left[i \mathcal{O}_{t \bar{t} \rightarrow F}^{(l)}(x) i \mathcal{O}_{g g \rightarrow t \bar{t}}^{(k)}(0)\right]\right| g g\right\rangle_{\mathrm{EFT}} \\
+C_{g g \rightarrow F}\left\langle F\left|i \mathcal{O}_{g g \rightarrow F}(0)\right| g g\right\rangle_{\mathrm{EFT}},
\end{aligned}
$$

where the matrix elements have to be evaluated in the EFT. In analogy with [51, 52] we call the contributions in the first and second line of (3.1) line the 'resonant' and 'non-resonant' amplitude, respectively. This structure is shown in figure 5 in diagrammatic form.

The 'resonant' part in the first line of (3.1) contains the contributions that involve a non-relativistic top quark pair, i.e. a top pair that is close to being on resonance. This entails that only a soft spatial momentum can be exchanged between the initial and final state. Since the incoming gluons contain hard momentum components they must be connected by a hard subgraph. The same holds for the two final state particles. Integrating out these hard subgraphs yields local production operators

$$
\left[\mathcal{O}_{g g \rightarrow t \bar{t}}^{(k)}\right]^{\mu \nu}=\mathcal{A}_{c}^{\perp \mu} \mathcal{A}_{\bar{c}}^{\perp \nu} \psi^{\dagger} \Gamma^{(k)} \chi
$$

that annihilate the incoming gluons and create a non-relativistic top pair and local annihilation operators

$$
\mathcal{O}_{t \bar{t} \rightarrow F}^{(l)}=\chi^{\dagger} \Gamma^{(l)} \psi \phi_{F}^{\dagger}
$$



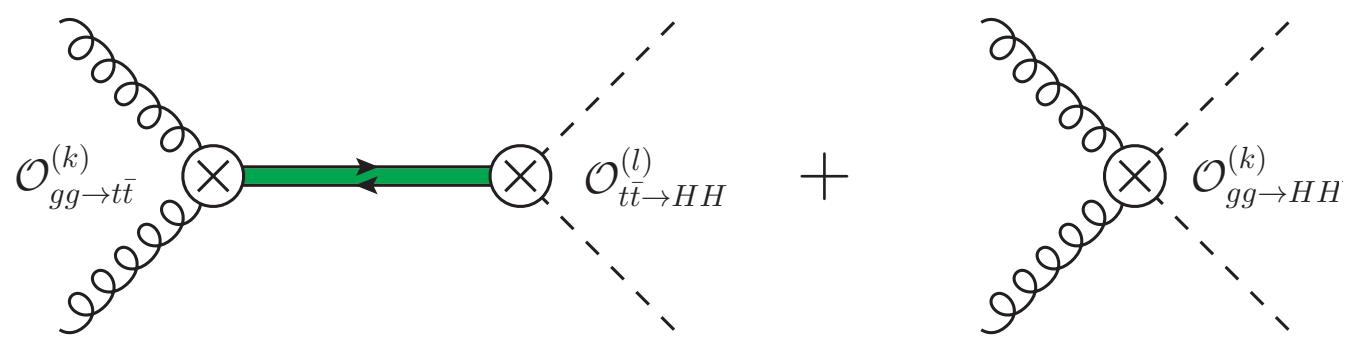

Figure 5. Graphical representation of the terms in the master formula (3.1). The diagram on the left (right) corresponds to the 'resonant' ('non-resonant') part of the amplitude. The shaded area indicates that Coulomb exchanges between the top quark pair are resummed.

that annihilate the top pair and create the final-state particles. Here $\mathcal{A}_{\bar{c}}^{\perp}$ is the collinear gluon field given in [54], the non-relativistic two-component spinor fields $\psi$ and $\chi$ annihilate a top quark and produce an anti-top quark respectively, $\Gamma^{(k)}$ contains a combination of Pauli matrices, $\mathrm{SU}(3)_{c}$ generators and potentially covariant derivatives and $\phi_{F}^{\dagger}$ represents a combination of fields that produces the final state. Both types of operators have associated hard-matching coefficients that absorb the higher-order corrections from hard modes. The propagation of the non-relativistic top pair is subject to a non-local color Coulomb interaction that manifests as $\alpha_{s} / \sqrt{1-z}$ corrections in the amplitude. These so-called Coulomb singularities can be resummed to all orders within PNRQCD. The 'resonant' contribution contains non-analytic $\sqrt{1-z}$ and $\ln (1-z)$ terms that correspond to on-shell cuts of the non-relativistic top pair.

Contributions where a hard momentum component is exchanged between the initial and the final state are contained in the 'non-resonant' part in the second line of (3.1). In the EFT they are represented by the matrix element of the local operator

$$
\left[\mathcal{O}_{g g \rightarrow F}\right]^{\mu \nu}=\mathcal{A}_{c}^{\perp \mu} \mathcal{A}_{\bar{c}}^{\perp \nu} \phi_{F}^{\dagger},
$$

that annihilates the incoming state and creates the final state. Since the top quarks cannot be on shell near threshold when they carry hard momentum, there are no discontinuities associated with $t \bar{t}$ cuts. Therefore, this contribution admits the form of a Taylor expansion in $(1-z)$ once massless cuts have been separated as described in section 2.1. The computation of this contribution is very involved since already the leading term in the Taylor expansion has the complexity of the full amplitude evaluated directly at the threshold $z=1$. However, we expect the Padé approximation to predict this unknown analytic part of the amplitude very accurately, even when using only the LME as input. Indeed, as we showed explicitly in section 2.1, adding the knowledge of just the non-analytic terms near threshold is already sufficient to reconstruct the full top-quark mass dependence with high accuracy. Therefore we can safely ignore the non-resonant contribution and only focus on the much simpler factorizable part. 


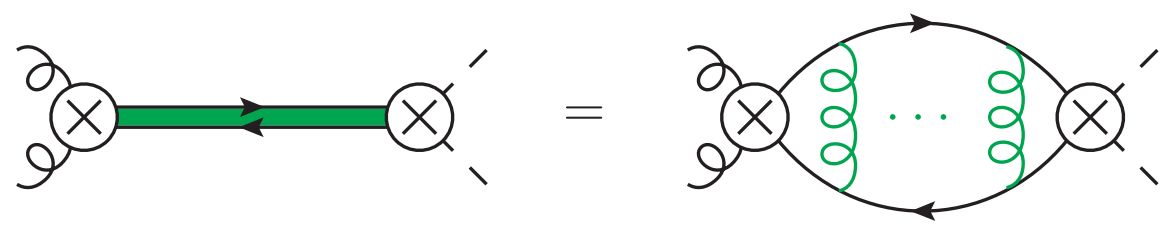

Figure 6. Matrix element at leading order in the power counting $\alpha_{s} \sim \sqrt{1-z}$.

\subsection{Computation of the non-analytic terms}

In this section we describe the computation of the 'resonant' part of the amplitude (3.1). We adopt here the non-relativistic power counting where $\alpha_{s} \sim \sqrt{1-z}$ and denote the $k^{\prime}$ th order in this counting by $\mathrm{nrN}^{k} \mathrm{LO}$ to distinguish it from the fixed-order expansion in the strong coupling constant. At nrLO, the matrix element is given by a non-relativistic Green function which resums the $1 / \sqrt{1-z}$ enhanced effects from the ladder-exchange of Coulomb gluons as indicated in figure 6. Hence, at any loop order, the leading non-analytic term in the threshold expansion of the amplitude can be determined by expanding the nrLO result to the respective order in $\alpha_{s}$. Up to nrNNLO, terms of the relative order

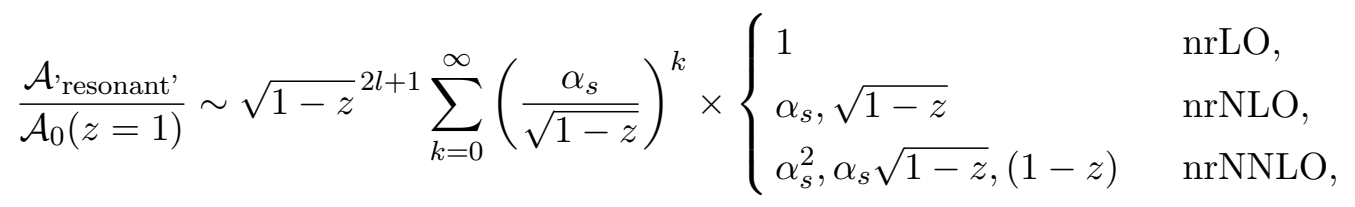

must be included, where $\mathcal{A}_{0}(z=1)$ is the LO amplitude evaluated at the top threshold, $l=0,1, \ldots$ denotes the angular momentum of the top pair and the global factor $\sqrt{1-z}$ accounts for the suppression of the phase-space near threshold.

Figure 7 illustrates the relation between different orders in standard relativistic perturbation theory and in the non-relativistic effective theory. For example, the following terms on the right-hand side of eq. (3.5) contribute to the fixed-order expansion up to NLO:

- The nrLO terms with relative factors $\sqrt{1-z}^{2 l+1}, \alpha_{s} \sqrt{1-z}^{2 l}$.

- The nrNLO terms with relative factors $\sqrt{1-z}^{2 l+2}, \alpha_{s} \sqrt{1-z}^{2 l+1}$.

- The nrNNLO terms with relative factors $\sqrt{1-z}^{2 l+3}, \alpha_{s} \sqrt{1-z}^{2 l+2}$.

For the processes $g g \rightarrow H^{(*)}$ and $g g \rightarrow H H$ there is no contribution from S-wave $t \bar{t}$ states due to parity and C-parity conservation. ${ }^{4}$ The leading 'resonant' contribution therefore contains the $\mathrm{P}$-wave Green function [76] which is suppressed by $(1-z)$ near threshold. We want to determine the 'resonant' amplitude up to nrNLO in the scaling (3.5), which contains the next-to-leading non-analytic terms in the threshold expansion at any loop order. In addition we compute the first two terms in the fixed-order expansion of the nrNNLO result in $\alpha_{s}$, i.e. those of relative orders $(1-z)^{5 / 2}$ and $\alpha_{s}(1-z)^{2}$. They correspond

\footnotetext{
${ }^{4}$ The $H$ and $H H$ final states have even parity and C-parity and the $t \bar{t}$ state with angular momentum $l$ and spin $s=0,1$ has $P=(-1)^{l+1}$ and $C=(-1)^{l+s}$. Thus, $l$ is one $(H)$ or odd $(H H)$ and $s=1$.
} 


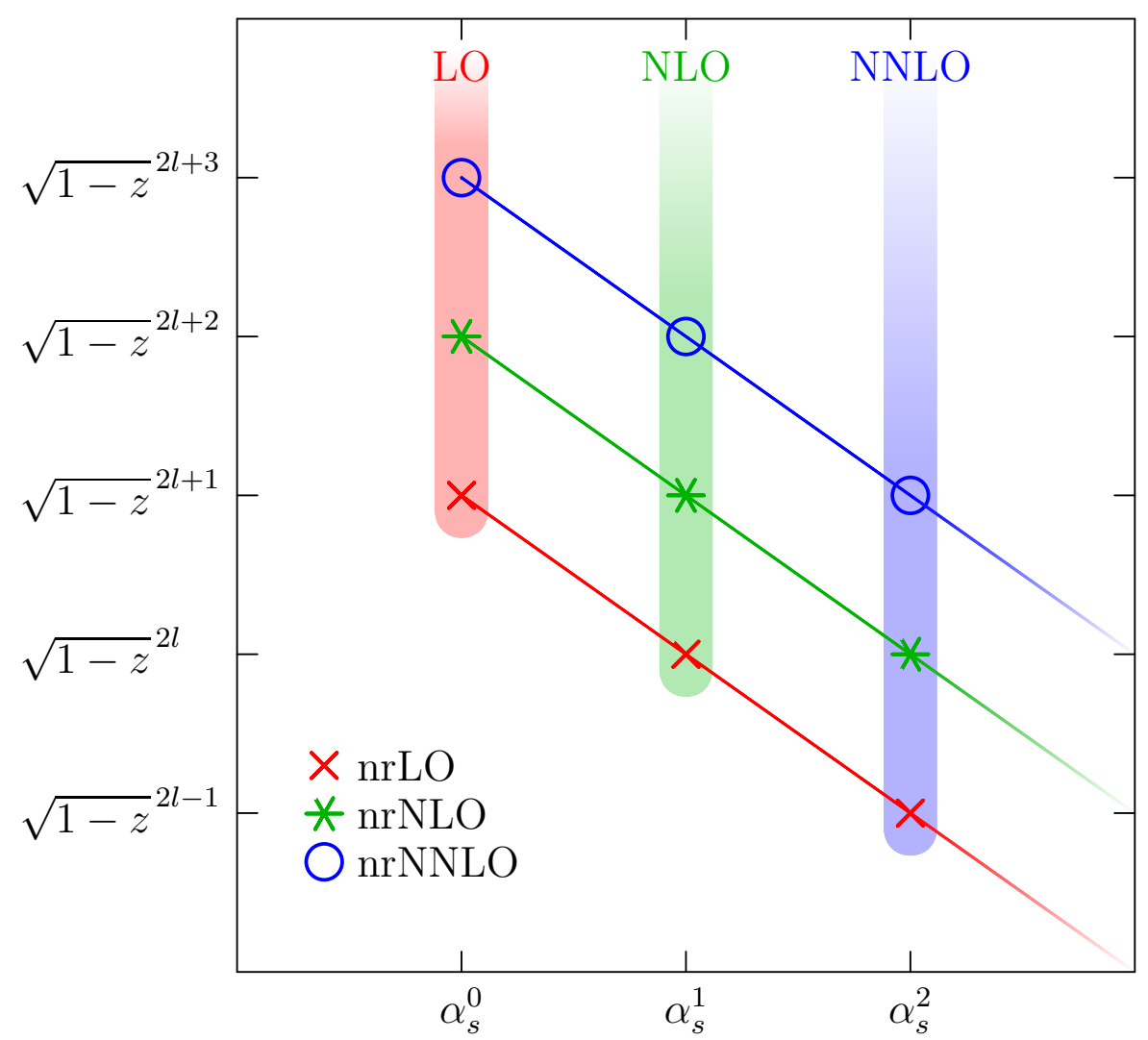

Figure 7. Relation between relativistic (LO, NLO, NNLO) and non-relativistic (nrLO, nrNLO, nrNNLO) power counting up to next-to-next-to-leading order. The axes show the powers of $\alpha_{s}$ and $\sqrt{1-z}$ in the various coefficients represented by the markers. Note that the normalization is chosen such that $\alpha_{s}^{0}$ corresponds to LO.

to the next-to-next-to leading threshold terms for the one and two loop amplitude which we study in section 4 .

The matrix elements in (3.1) receive corrections from the higher-order non-local potentials and the dynamical modes contained in the EFT. The EFT contains no interactions of collinear modes with non-relativistic modes or between collinear modes of different directions. They cannot be present because the combination of the involved momenta yields hard modes which have been integrated out. Therefore the only collinear corrections at nrNLO are from the left diagram in figure 8. The corresponding loop integral is scaleless and therefore vanishes in dimensional regularization.

Ultrasoft gluons couple to the collinear and non-relativistic sector as well as to the $\mathrm{P}$-wave production and annihilation operators. The exchange of ultrasoft gluons between the collinear states shown in the diagram on the right of figure 8 yields only scaleless integrals. The interactions in the EFT must be multipole expanded. At leading order in the multipole expansion ultrasoft gluons couple to the net color charge of the $t \bar{t}$ state since the large wavelength $\lambda \sim 1 /\left(m_{t}(1-z)\right)$ gluons cannot resolve the spatial separation $a_{\mathrm{B}} \sim 1 /\left(m_{t} \sqrt{1-z}\right)$ of the top pair. The first non-vanishing term in the multipole expansion 

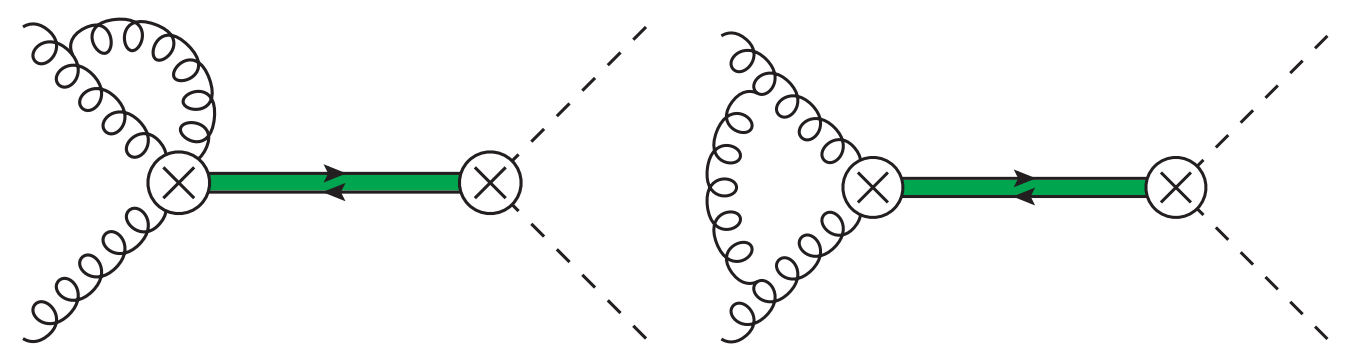

Figure 8. nrNLO diagrams involving collinear (left) and ultrasoft (right) gluon radiation. Both loop integrals are scaleless and vanish in dimensional regularization.

for color singlet states is therefore the chromoelectric term $\psi^{\dagger} \mathbf{x} \cdot \mathbf{E} \psi$ which is suppressed by $\alpha_{s}^{1 / 2} \sqrt{1-z} \sim \alpha_{s}^{3 / 2}$. Similarly the ultrasoft gluon term in the covariant derivative in the $\mathrm{P}$-wave operators is suppressed by $\alpha_{s}^{1 / 2} \sqrt{1-z} \sim \alpha_{s}^{3 / 2}$ with respect to the derivative term. A single insertion of either of these subleading terms vanishes by rotational invariance [55]. Thus, contributions from the subleading interactions require at least two insertions and first appear at nrNNNLO.

The effects of higher-order potentials enter as corrections to the non-relativistic Green function. The nrNNNLO S-wave and nrNLO P-wave Green functions have been computed for $t \bar{t}$ production in $e^{+} e^{-}$collisions near threshold [76, 77]. We determine the $\alpha_{s}^{0,1}$ terms in the nrNNLO P-wave Green function in appendix B. Up to the considered order the resonant amplitudes hence take the simple factorized form

$$
\mathcal{A}_{\text {resonant }}=\sum_{k, l} \mathcal{N}_{k l}(1-z) C_{g g \rightarrow t \bar{t}}^{(k)} C_{t \bar{t} \rightarrow F}^{(l)} G_{S, P}(1-z) .
$$

The Wilson coefficients $C_{g g \rightarrow t \bar{t}}^{(k)}, C_{t \bar{t} \rightarrow F}^{(l)}$ are perturbative in $\alpha_{s}$ and independent of $z$. We can compute them via matching to the full Standard Model, i.e. by performing a Taylor expansion of the on-shell amplitudes for $g g \rightarrow t \bar{t}, t \bar{t} \rightarrow F$ around the top threshold and comparing to the matrix elements of the effective operators $\mathcal{O}_{g g \rightarrow t \bar{t}}^{(k)}, \mathcal{O}_{t \bar{t} \rightarrow F}^{(l)}$. Subleading terms in the Taylor expansion in $(1-z)$ correspond to higher-dimensional operators, which contain derivatives acting on the non-relativistic top and anti-top fields. Since $(1-z) \sim \alpha_{s}^{2}$, we only require matrix elements with at most one subleading operator up to nrNNLO. The normalization factors $\mathcal{N}_{k l}$ are either $z$-independent, if the operators $\mathcal{O}_{g g \rightarrow t \bar{t}}^{(k)}$ and $\mathcal{O}_{t \bar{t} \rightarrow F}^{(l)}$ are of leading order in the non-relativistic expansion, or proportional to $(1-z) \sim \alpha_{s}^{2}$, if one of the operators is of subleading order. To achieve the accuracies specified in (3.5) we require the following ingredients

- $\operatorname{nrLO}$ :

- the tree-level coefficients $C_{g g \rightarrow t \bar{t}}^{(k)}, C_{t \bar{t} \rightarrow F}^{(l)}$

- the nrLO Green function $G_{S, P}(1-z)$ 
- nrNLO: the above and

- the one-loop coefficients $C_{g g \rightarrow t \bar{t}}^{(k)}, C_{t \bar{t} \rightarrow F}^{(l)}$

- the nrNLO Green function $G_{S, P}(1-z)$

- the order $\alpha_{s}^{0,1}$ terms at nrNNLO: the above and

- the tree-level coefficients $C_{g g \rightarrow t \bar{t}}^{(k)}, C_{t \bar{t} \rightarrow F}^{(l)}$ for the $(1-z)$-suppressed operators

- the $\alpha_{s}^{0,1}$ terms in the nrNNLO Green function $G_{S, P}(1-z)$

- nrNNLO: the above and

- the two-loop coefficients $C_{g g \rightarrow t \bar{t}}^{(k)}, C_{t \bar{t} \rightarrow F}^{(l)}$

- the nrNNLO Green function $G_{S, P}(1-z)$

As mentioned before, it is sufficient to know the nrNNLO terms proportional to $\alpha_{s}^{0}$ and $\alpha_{s}^{1}$ in order to construct approximations to two-loop (NLO) fixed-order amplitudes (cf. figure 7). The remaining nrNNLO terms of the relative order $\alpha_{s}^{2}(1-z)^{3 / 2}$ will be important for the reconstruction of the three-loop amplitude. Since its determination requires the calculation of the two-loop matching coefficients $C_{g g \rightarrow t \bar{t}}^{(k)}$ and $C_{t \bar{t} \rightarrow F}^{(l)}$ as the most complicated ingredient, we postpone this to future work.

The one-loop coefficients $C_{t \bar{t} \rightarrow F}^{(l)}$ are finite after field and mass renormalization. The one-loop coefficients $C_{g g \rightarrow t \bar{t}}^{(k)}$, however, require additional IR subtractions since the virtual amplitude by itself is not IR safe. Our results for the threshold expansion of the form factors are given in (2.8)-(2.11) and appendix $\mathrm{C}$ together with the details of the IR subtractions. Together with the nrNLO expression for the P-wave Green function [76] these results are sufficient to determine the leading and next-to-leading non-analytic terms in the threshold expansion of the form factors at any order in $\alpha_{s}$.

Another interesting, yet more involved, application of our formalism is Higgs plus jet production. Here, we shortly comment on that, but leave a more careful assessment to future work. The amplitudes $g g \rightarrow H g, g q \rightarrow H q$ and $q \bar{q} \rightarrow H g$ obey the same structure of (3.1) near the top threshold but the corresponding 'resonant' matrix elements are more complicated since the final state now contains a color-charged particle. Ultrasoft gluons can then be exchanged between the initial state, the final state and the intermediate top pair which is in a color octet state and no longer decouples. In [53-55] it was demonstrated for arbitrary color structures that the 'resonant' matrix elements in forward-scattering amplitudes factorize into the convolution of a non-relativistic Green function, therein called the potential function, and an ultrasoft function, therein called the soft function. At leading power this follows from field transformations that decouple the collinear and non-relativistic fields from the ultrasoft fields. The extension to higher orders requires a careful assessment of the subleading interactions and was performed to NNLL in [53-55]. Following these derivations we identified no aspect that would obstruct the extension to Higgs plus jet production and therefore conjecture that an analogous factorization formula holds for the corresponding amplitudes. 


\section{Comparison with the exact result}

As a proof of method, we compare our results at LO and NLO with the results in full top mass dependence for Higgs pair production. While at LO, the Higgs pair production cross section is known in full mass dependence since the late 80's [78-80], the computation of the NLO QCD corrections is quite involved, due to the many scales of the problem. The first work on the NLO corrections was based on the heavy top mass limit [81] reweighted with the matrix elements squared of the full LO results (HEFT). The real corrections in full top mass dependence have been computed in $[82,83]$, while the virtual corrections have been kept in HEFT. The computation of the virtual corrections in full top mass dependence became available only recently in $[9,10]$.

\subsection{Numerical setup}

For the numerical evaluation we choose a centre-of-mass energy of $\sqrt{s}=14 \mathrm{TeV}$. The Higgs boson mass has been set equal to $m_{H}=125 \mathrm{GeV}$ and the top quark mass to $m_{t}=173 \mathrm{GeV}$. We do not account for bottom quark loops as they contribute with less than $1 \%$ at LO. We have adopted the PDF set NNPDF3.0 [84]. The strong coupling constant is set to $\alpha_{s}\left(M_{Z}\right)=0.118$ at LO and NLO. The renormalization scale has been set to $M_{H H} / 2$, where $M_{H H}$ denotes the invariant mass of the Higgs boson pair, as suggested by the NNLL soft gluon resummation performed in $[85,86]$.

We construct our Padé approximants at LO (NLO) as described in section 2 by solving numerically the 8 (7) equations from the LME [24] and threshold expansion, given in section 2.1 and appendix C, by means of the FORTRAN routine MINPACK $[87,88] .{ }^{5}$ For every phase space point we construct a total of 100 Padé approximants $[n / m]$, where $a_{R}$ takes a random value between $[0.1,10], n, m \in[1,6]$ at $\mathrm{LO}$ and $n, m \in[1,5]$ at NLO, and take the mean value. From that we obtain an error estimate on every form factor by taking the standard deviation. For the computation of the cross section or the virtual corrections we add up the errors stemming from the different form factors quadratically. Padé approximants with poles in $\operatorname{Re}(z) \in[0,8]$ and $\operatorname{Im}(z) \in[-1,1]$ were excluded, since functions with poles close-by in the complex plane could have an unwanted resonant behaviour. The running time per phase space point for the construction of 100 Padé approximants at NLO is usually below $6 \mathrm{~s}$.

\subsection{Comparison at $\mathrm{LO}$}

In table 1 we give the results for the $\mathrm{LO}$ cross section in different approximations. The first row, $[n / m]$ w/o THR, symbolizes the cross section obtained with Padé approximants constructed without input from the threshold expansion, where $n, m \in[1,3]$ and approximants with poles as described above have been excluded. The result we obtain when including the threshold information and using the specifications described in section 4.1 is denoted by $[n / m]$. With $[n / n \pm 1,3]$ we symbolize the results we find when only the Padé approximants

\footnotetext{
${ }^{5}$ We provide a FORTRAN routine of the Padé approximated matrix elements upon request.
} 


\begin{tabular}{|cc|}
\hline & $\sigma[\mathrm{fb}]$ \\
\hline$[n / m]$ w/o THR & $19.9 \pm 5.4$ \\
{$[n / m]$} & $21.7 \pm 1.1$ \\
{$[n / n \pm 1,3]$} & $21.3 \pm 0.4$ \\
full & 21.3 \\
\hline
\end{tabular}

Table 1. Numbers for the total LO cross section and standard deviation from the construction of 100 Padé approximants.

[5/2], [4/3], [3/4] and [2/5] are used. ${ }^{6}$ Finally, we give the full LO cross section (obtained with HPAIR [89]) in the fourth row of table 1 . As can be inferred from the table, the Padé approximants provide a very good approximation for the full cross section, in particular if only the most diagonal and next-to-diagonal Padé approximants are constructed. The threshold expansion proves to be essential for a good approximation. As expected, the standard deviation computed from the construction of $100[n / m]$ Padé approximants with random $a_{R}$ and different $n, m$ becomes smaller if we construct only the most diagonal and next-to-diagonal Padé approximants.

In figure 9 we show the invariant Higgs mass distribution for the full result (dark blue), the $[n / n \pm 1,3]$ Padé approximants (pink) and the Padé approximants without the threshold expansion (light blue). While the $[n / n \pm 1,3]$ full Padé approximants fit the shape of the invariant mass distribution in full mass dependence almost perfectly, the approximation where the threshold expansion is not included (hence the approximation is only built from the LME) fits the shape only for small invariant mass. The error on the construction of the approximation including the threshold expansion is rather small whereas if the approximation is constructed only from the LME, the error becomes much larger in particular above the threshold.

We thus conclude that at LO our approximation of the mass effects by Padé approximants works well as long as the conditions obtained from the threshold expansion are included. Using only nearly diagonal Padé approximants leads to a result with smaller error with values closer to the true result.

\subsection{Comparison at NLO}

Finally, we compare our results to the computation of the NLO corrections in full top mass dependence of refs. [9, 10]. In the framework of ref. [90] a grid and an interpolation function with numerical values for the virtual corrections of refs. [9, 10] have been provided.

\footnotetext{
${ }^{6}$ Note however that these are mainly [5/2] and [4/3] Padé approximants as [3/4] and [2/5] usually are excluded by our pole criterion.
} 


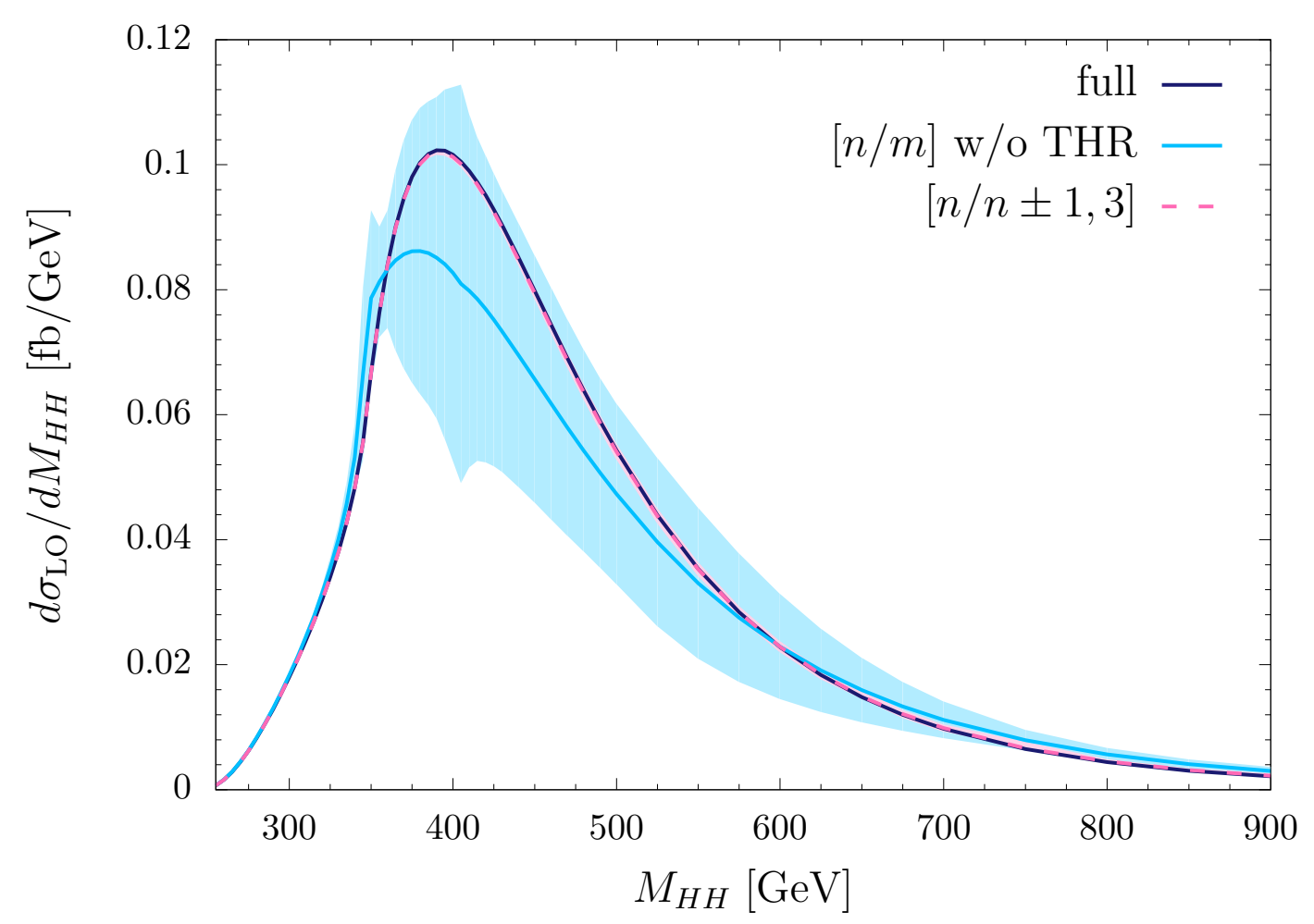

Figure 9. Invariant Higgs mass distribution for the full LO cross section (dark blue), the $[n / n \pm 1,3]$ Padé approximants (pink line) and the Padé constructed without threshold expansion (light blue). The standard deviation of the Padé lines are shown by the semi-transparent regions with the corresponding color. The pink band is barely wider than the width of the curves and hardly visible.

In order to fit the conventions of ref. [90] we define the finite part of the virtual corrections as

$$
\begin{aligned}
\mathcal{V}_{\text {fin }} & =\frac{\alpha_{s}^{2}\left(\mu_{R}\right)}{16 \pi^{2}} \frac{\hat{s}^{2}}{128 v^{4}}\left[\left|\mathcal{M}_{\text {born }}\right|^{2}\left(C_{A} \pi^{2}-C_{A} \log ^{2}\left(\frac{\mu_{R}^{2}}{\hat{s}}\right)\right)\right. \\
& \left.+2\left\{\left(F_{1}^{1 l}\right)^{*}\left(F_{1}^{2 l,[n / m]}+F_{1}^{2 \Delta}\right)+\left(F_{2}^{1 l}\right)^{*}\left(F_{2}^{2 l,[n / m]}+F_{2}^{2 \Delta}\right)+\text { h.c. }\right\}\right]
\end{aligned}
$$

with

$$
\left|\mathcal{M}_{\text {born }}\right|^{2}=\left|F_{1}^{1 l}\right|^{2}+\left|F_{2}^{1 l}\right|^{2}
$$

and $F_{1}$ defined in eq. (C.2). For $F_{x}^{2 l,[n / m]}$ we use the matrix elements constructed with the Padé approximant $[n / m]_{\tilde{f}}$. All other matrix elements are used in full top mass dependence. The form factors $F_{x}^{2 \Delta}$ stem from the double triangle contribution to the virtual corrections and can be expressed in terms of one-loop integrals. They are given in ref. [24] in full top mass dependence. In the heavy top mass limit they become

$$
F_{1}^{2 \Delta} \rightarrow \frac{4}{9}, \quad F_{2}^{2 \Delta} \rightarrow-\frac{4}{9} \frac{p_{T}^{2}}{2 \hat{t} \hat{u}}\left(\hat{s}-2 m_{H}^{2}\right)
$$




\begin{tabular}{|cccccc|}
\hline & & \multicolumn{4}{c|}{$\mathcal{V}_{\text {fin }} \times 10^{4}$} \\
\cline { 3 - 6 }$M_{H H}[\mathrm{GeV}]$ & $p_{T}[\mathrm{GeV}]$ & HEFT & {$[n / m]$} & {$[n / n \pm 0,2]$} & full \\
\hline 336.85 & 37.75 & 0.912 & $0.996 \pm 0.004$ & $0.990 \pm 0.001$ & $0.996 \pm 0.000$ \\
350.04 & 118.65 & 1.589 & $1.933 \pm 0.012$ & $1.937 \pm 0.010$ & $1.939 \pm 0.061$ \\
411.36 & 163.21 & 4.894 & $4.326 \pm 0.183$ & $4.527 \pm 0.069$ & $4.510 \pm 0.124$ \\
454.69 & 126.69 & 6.240 & $5.300 \pm 0.192$ & $5.114 \pm 0.051$ & $5.086 \pm 0.060$ \\
586.96 & 219.87 & 7.797 & $4.935 \pm 0.583$ & $5.361 \pm 0.281$ & $4.943 \pm 0.057$ \\
663.51 & 94.55 & 8.551 & $5.104 \pm 1.010$ & $4.096 \pm 0.401$ & $4.120 \pm 0.018$ \\
\hline
\end{tabular}

Table 2. Numbers for the virtual corrections for some representative phase space points for the HEFT result reweighted with the full Born cross section (as in ref. [81]), the Padé-approximated ones and the full calculation [90].

The contribution of the double triangle diagrams to the virtual corrections is only of the order of a few per cent [91].

In table 2 we compare values for the full computation of the virtual corrections obtained from the grid of ref. [90], the HEFT results rescaled with the full Born cross section (as e.g. implemented in HPAIR), and the Padé approximations including all possible approximants without poles in $\operatorname{Re}(z) \in[0,8]$ and $\operatorname{Im}(z) \in[-1,1]$ (called $[n / m]$ ) and the ones where we only construct diagonal [3/3] and next-to diagonal [4/2] and [2/4] approximants (called $[n / n \pm 0,2]$ ). The errors given in the table are, in case of the Padé-approximated results, due to the construction of the different approximants and due to the rescaling with $a_{R}$. For the full results the error stems from internal binning in the grid. As can be inferred from the table, the Padé construction approximates the full result quite well. It provides a much better approximation than the HEFT results with a generally reliable error estimate. While up to $M_{H H}=450 \mathrm{GeV}$ the Padé method provides an excellent approximation on the level of $\lesssim 2 \%$, for larger invariant masses and $p_{T}$ the results worsen gradually. As already anticipated from the LO results, constructing only diagonal and next-to diagonal Padé approximants improves both the error and the values of the virtual corrections with respect to the full result. Indeed we even find that only constructing diagonal Padé approximants gives results even closer to the full result. Since this does not allow for a reliable error estimate any more (the error would then solely stem from the variation of $a_{R}$ ) we do not discuss this here any further.

In figure 10 we show for $p_{T}=100 \mathrm{GeV}$ the virtual corrections $\mathcal{V}_{\text {fin }}$ for varying $M_{H H}$ for the Padé approximations $[n / n \pm 0,2]$, the Padé approximants constructed only from the LME, the full result and the reweighted HEFT results. Again, we can see that contrary to the HEFT results the Padé approximation can reproduce the correct scaling with the invariant mass of the full result. The quality of the approximation is improved significantly with the inclusion of the threshold expansion. The error of the Padé approximation increases with the invariant mass. Note that the full result has, apart from the previous error from the internal binning, also an error due to the interpolation procedure. We do 


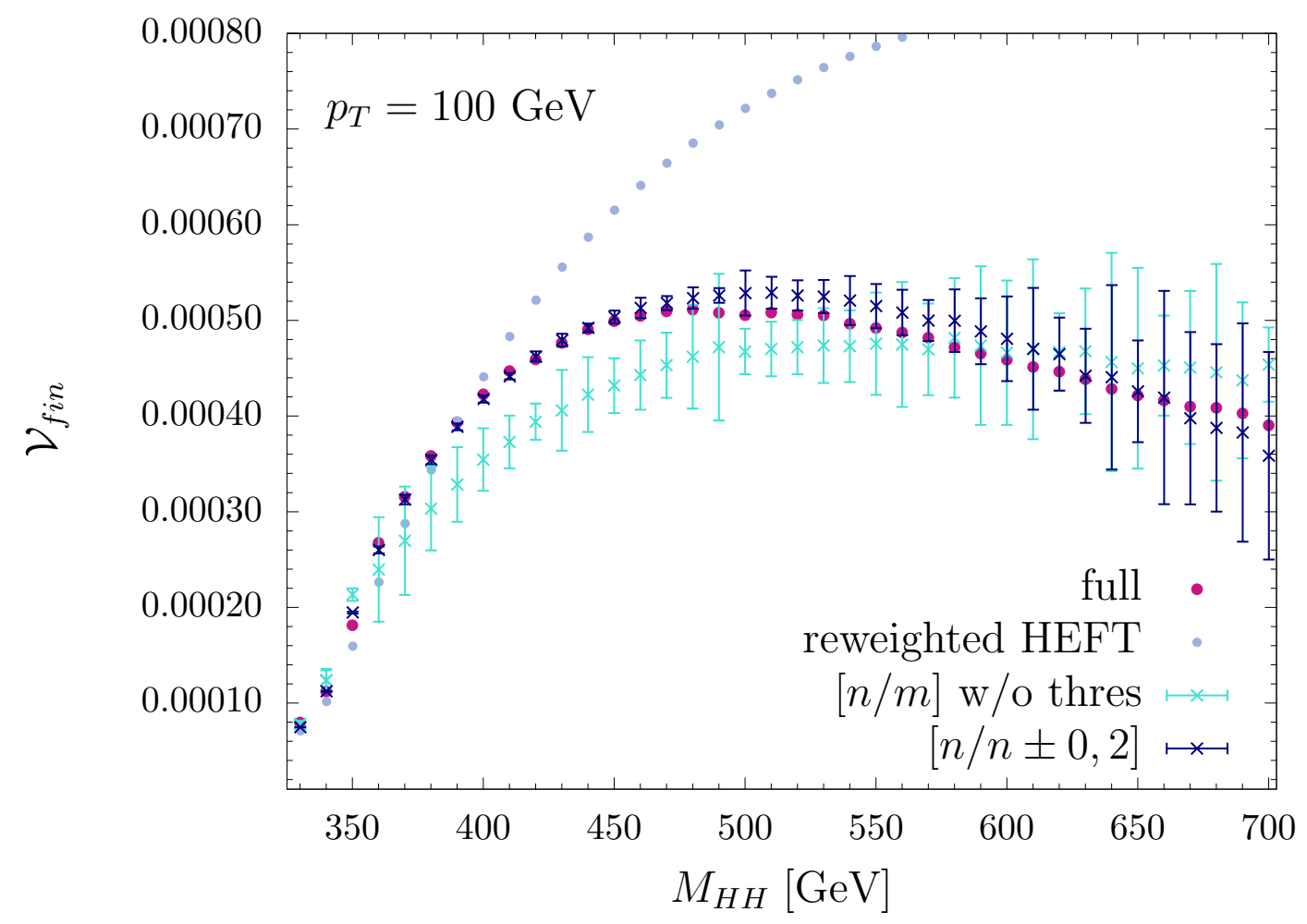

Figure 10. Finite part of the virtual corrections, $\mathcal{V}_{\text {fin }}$, as a function of $M_{H H}$ for $p_{T}=100 \mathrm{GeV}$. The light blue points are the reweighted HEFT results, the pink points the virtual corrections in full top mass dependence from the interpolation function provided with ref. [90], the dark blue points are from the diagonal and off-diagonal Padé approximants with their standard deviation and the turquoise points with standard deviation are the Padé approximants constructed without the threshold expansion.

not quantify this error but in comparison to the HEFT grid provided with ref. [90] we conclude that while in the range up to $M_{H H} \lesssim 570 \mathrm{GeV}$ this error is negligible, it will be a few $\%$ for larger $M_{H H}$. The comparison with the numerical results of [90] demonstrates that our prescription for the uncertainty related to the construction of Padé approximants also provides a reasonable error estimate at NLO.

In conclusion, we see that for the NLO corrections the Padé approximation reproduces the correct scaling behaviour for small and moderate invariant mass and $p_{T}$. Since the cross section peaks around $M_{H H} \approx 400 \mathrm{GeV}$ and $p_{T} \approx 150 \mathrm{GeV}$ this will lead to a reliable approximation and reliable error estimate also for the full cross section. It can be expected that both the error and the difference with respect to the full result improves once more input is used (i.e. higher orders in the threshold expansion, higher orders in the LME, possibly input from a small mass expansion).

\section{Conclusions and outlook}

We have reconstructed the top-quark mass dependence of the one and two loop virtual amplitudes for Higgs pair production in gluon fusion with Padé approximants based on 
the LME of the amplitude [24] and new analytic results near the top threshold $\hat{s}=4 m_{t}^{2}$. We observe perfect agreement of the one-loop results with the exact expressions once the additional conditions from the threshold terms are imposed. Significant deviations are observed when only the LME is used to construct Padé approximants, but we still find agreement within the uncertainty estimate of our reconstruction, which is based on variation of the rescaling parameter $a_{R}$ and the use of different $[n / m]$ approximants. At the two-loop level the full result can be reproduced in the entire phenomenologically relevant range within typical uncertainties ranging from below $\pm 3 \%$ in the region $M_{H H} \leq 450 \mathrm{GeV}$ up to about $\pm 20 \%$ for $M_{H H}=700 \mathrm{GeV}$. Thus, our method allows for a determination of the total cross section including top-quark mass effects at NLO where the uncertainty due to the reconstruction is negligible compared to the scale uncertainty which is of the size of $\pm 13 \%[9,10]$. This represents considerable progress compared to the rescaled HEFT and LME approximations where a reliable uncertainty estimate is not possible. Our method can also be systematically improved by including higher orders in the LME or threshold expansions. We expect even better behaviour if one also considers the leading term in the small-mass expansion $z \rightarrow \infty$ which corresponds to the bottom-quark contribution expanded for small $m_{b}$. An approach for computations in this limit has recently been introduced [49, 50, 92]. Furthermore our results strongly suggest that the combination of the Padé approximants of the NLO virtual corrections with the exact evaluation of the real corrections $[82,83]$ can reproduce differential distributions to high accuracy.

There is a large number of possible applications for our method. To further increase the precision for Higgs pair production one needs to consider NNLO QCD corrections. The rescaled HEFT approximation for the NNLO corrections increases the cross section by $18 \%$ [10] which exceeds the estimate from scale variation at NLO. A NNLO computation which retains the full top-quark mass effects is clearly out of reach of the current technology. On the other hand, the LME has already been computed up to $1 / m_{t}^{4}$ in [23] and we have determined the two first non-analytic terms in the threshold expansion. This presently available input only allows for the construction of Padé approximants with $n+m=3$ where we do not expect stable behaviour, but a calculation of two or three more expansion parameters would allow the evaluation of NNLO corrections in the soft-virtual approximation of $[23,93]$. Additionally, one can study the NLO electroweak corrections involving topquark loops. Of particular interest are the contributions involving additional Higgs bosons which alter the dependence of the cross section on the values of the Higgs self couplings.

It is straightforward to apply our method to $g g \rightarrow H Z$ and the top-quark mediated $g g \rightarrow Z Z$ amplitude and at higher orders in perturbation theory. In all these cases, results in the LME have been obtained at two loops [25-27] and for $g g \rightarrow H^{(*)}$ even at three loops [18-22]. The determination of the threshold terms only requires the computation of the respective one-loop matching coefficients in (3.6). Another phenomenologically very interesting case is Higgs plus jet production. The construction of Padé approximants is also possible here but the computation of the threshold expansion is more involved as we outlined in section 3.2. Beyond LME results, also the leading term in the small-mass expansion is know for the relevant two-loop amplitudes $[49,50]$. Hence, the effects of this additional input on the reconstruction of top-quark mass effects can be studied in this case. 


\section{Acknowledgments}

We thank Johannes Schlenk for useful discussions and Matthias Kerner for clarifications regarding the grid of ref. [90]. RG acknowledges useful discussion with Fabrizio Caola, Keith Ellis and Sebastian Kirchner in an early stage of this project. RG and AM are supported by a European Union COFUND/Durham Junior Research Fellowship under the EU grant number 609412.

\section{A Subtractions}

We construct functions for the threshold subtractions based on the known analytical results for the current correlators. The subtraction functions and their threshold expansions are

$$
\begin{aligned}
& s_{1}(z)=\frac{2}{\pi}(1-z) G(z) \stackrel{z \rightarrow 1}{\asymp} \sqrt{1-z}+\frac{1}{2}(1-z)^{3 / 2}+\frac{3}{8}(1-z)^{5 / 2}+\mathcal{O}\left((1-z)^{7 / 2}\right), \\
& s_{2}(z)=-\frac{16(1-z) \Pi^{(1), v}(z)}{3 z} \\
& z \varlimsup^{\beth}(1-z) \ln (1-z)-\frac{8}{\pi}(1-z)^{3 / 2}+\frac{1}{3}(1-z)^{2} \ln (1-z)+\mathcal{O}\left((1-z)^{5 / 2}\right), \\
& s_{3}(z)=\frac{2}{\pi} \frac{(1-z)^{2} G(z)-1}{z} \stackrel{z \rightarrow 1}{\leftrightarrows}(1-z)^{3 / 2}+\frac{3}{2}(1-z)^{5 / 2}+\mathcal{O}\left((1-z)^{7 / 2}\right), \\
& s_{4}(z)=-\frac{8}{81 \pi^{2}} \frac{54 \pi^{2}(1-z)^{2} \Pi^{(1), v}(z)-41 z}{z^{2}} \stackrel{z \rightarrow 1}{\beth^{1}}(1-z)^{2} \ln (1-z)+\mathcal{O}\left((1-z)^{5 / 2}\right), \\
& s_{5}(z)=\frac{2}{3 \pi} \frac{3(1-z)^{3} G(z)+7 z-3}{z^{2}} \stackrel{z \rightarrow 1}{\asymp}(1-z)^{5 / 2}+\mathcal{O}\left((1-z)^{3}\right),
\end{aligned}
$$

where we have used the symbol $\asymp$ to denote that terms analytical in $(1-z)$ have been dropped on the right-hand side,

$$
G(z)=\frac{1}{2 z \sqrt{1-1 / z}} \ln \left(\frac{\sqrt{1-1 / z}-1}{\sqrt{1-1 / z}+1}\right),
$$

and $\Pi^{(1), v}$ is the well-known two-loop correction to the vacuum polarization [94] in the convention of [31]. The functions $s_{i}$ in (A.1) are constant as $z \rightarrow 0$ and only diverge logarithmically as $z \rightarrow \infty$.

\section{B Expansion of the P-wave Green function}

The P-wave Green function has been computed up to nrNLO in [76]. In addition we have determined the terms of order $\alpha_{s}^{0}$ and $\alpha_{s}^{1}$ in the nrNNLO correction. Those are given by the insertion of the 'kinetic potential' [69]

$$
V_{\text {kin }}\left(\mathbf{p}, \mathbf{p}^{\prime}\right)=-\frac{\mathbf{p}^{4}}{4 m_{t}^{3}}(2 \pi)^{d-1} \delta^{(d-1)}\left(\mathbf{p}-\mathbf{p}^{\prime}\right)
$$

and the $1 / m^{2}$ potential [69]

$$
V_{1 / m^{2}}\left(\mathbf{p}, \mathbf{p}^{\prime}\right)=-\frac{4 \pi \alpha_{s} C_{F}}{\mathbf{q}^{2}}\left[\mathcal{V}_{1 / m^{2}} \frac{\mathbf{q}^{2}}{m_{t}^{2}}+\mathcal{V}_{p} \frac{\mathbf{p}^{2}+\mathbf{p}^{\prime 2}}{2 m_{t}^{2}}\right]
$$


where $\mathbf{q}=\mathbf{p}-\mathbf{p}^{\prime}$, the term proportional to $\mathcal{V}_{1 / m^{2}}$ vanishes for the P-wave due to asymmetry under the integration over the spatial momentum components and $\mathcal{V}_{p}=1+\mathcal{O}\left(\alpha_{s}\right)$. Our result for the $\mathrm{P}$-wave Green function expanded in $\alpha_{s}$ and $(1-z)$ reads

$$
\begin{aligned}
G_{P}(1-z) \stackrel{z \rightarrow 1}{\leftrightarrows} \frac{m_{t}^{4}}{4 \pi}\left\{\left[(1-z)^{3 / 2}-\frac{1}{2}(1-z)^{5 / 2}+\mathcal{O}\left((1-z)^{7 / 2}\right)\right]\right. \\
+\alpha_{s} C_{F}\left[\frac{1}{2}(1-z) \ln (1-z)-(1-z)^{2} \ln (1-z)+\mathcal{O}\left((1-z)^{3}\right)\right] \\
+\alpha_{s}^{2} C_{F}\left[-C_{F} \frac{3+\pi^{2}}{12} \sqrt{1-z}\right. \\
-\frac{1-z}{16 \pi}\left(\beta_{0} \ln ^{2}(1-z)-2\left(a_{1}+2 \beta_{0}\right) \ln (1-z)\right) \\
\left.\left.+\mathcal{O}\left((1-z)^{3 / 2}\right)\right]+\mathcal{O}\left(\alpha_{s}^{3}\right)\right\}
\end{aligned}
$$

where

$$
\beta_{0}=\frac{11}{3} C_{A}-\frac{4}{3} T_{F} n_{l}, \quad a_{1}=\frac{31}{9} C_{A}-\frac{20}{9} T_{F} n_{l},
$$

and $\asymp$ again indicates that terms analytic in $(1-z)$ have been dropped.

\section{Results for the $g g \rightarrow H H$ form factors near threshold}

We give the results for the threshold expansion of the $g g \rightarrow H H$ form factors up to threeloop order. The expansion of the form factors $F_{1}$ and $F_{2}$ in the strong coupling constant takes the form

$$
F_{i}=F_{i}^{1 l}+\frac{\alpha_{s}}{\pi}\left[F_{i}^{2 l}+F_{i}^{2 \triangle}\right]+\left(\frac{\alpha_{s}}{\pi}\right)^{2} \widetilde{F}_{i}^{3 l}+\ldots, \quad i=1,2 .
$$

At the two-loop level the contributions $F_{i}^{2 \triangle}$ that involve two top-quark loops are known exactly [24] and have therefore been separated in (C.1). They will not be considered further because their threshold expansion does not contain any non-analytic terms. The form factor $F_{1}$ is further decomposed into a 'triangle' and 'box' contribution

$$
F_{1}^{i l}=\frac{3 m_{H}^{2}}{\hat{s}-m_{H}^{2}} F_{\triangle}^{i l}+F_{\square}^{i l}, \quad i=1,2,
$$

as indicated in figure 11. The contributions of the 'triangle' diagrams to the form factor $F_{2}$ vanish. As discussed in section 2 we make massless cuts explicit

$$
\begin{array}{ll}
F_{i}^{2 l} & =C_{F} F_{i, C_{F}}^{2 l}+C_{A}\left(F_{i, C_{A}}^{2 l}+F_{i, C_{A}, \ln }^{2 l} \ln (-4 z)\right), \\
\widetilde{F}_{i}^{3 l} & =F_{i}^{3 l}+F_{i, \ln }^{3 l} \ln (-4 z)+F_{i, \ln ^{2}}^{3 l} \ln (-4 z)^{2},
\end{array}
$$

such that all the F's on the right-hand side of (C.3) and (C.4) are analytic in $z$ except for a branch cut for real $z \geq 1$. 


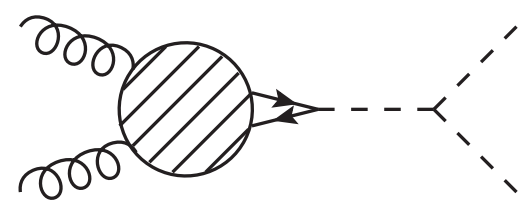

'Triangle' topology

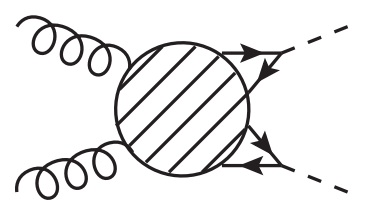

'Box' topology

Figure 11. The Feynman diagrams are divided into 'triangle' and 'box' topologies depending on whether they contain an intermediate s-channel Higgs boson or not.

Like the previous works $[5,6,24]$ we use the following $\overline{\mathrm{MS}}$ scheme convention

$$
\int d^{4} l \rightarrow \frac{\Gamma(1-\epsilon)}{(4 \pi)^{\epsilon}} \mu_{R}^{2 \epsilon} \int d^{d} l=\left[1+\frac{\pi^{2}}{12} \epsilon^{2}+\ldots\right] \frac{e^{\epsilon \gamma_{E}}}{(4 \pi)^{\epsilon}} \mu_{R}^{2 \epsilon} \int d^{d} l
$$

in our calculation. The renormalized form factors still contain IR divergences which cancel with contributions involving unresolved real radiation that are not considered here. We use subtractions of a minimal type as refs. $[5,6,24]^{7}$

$$
\begin{aligned}
& F_{i}^{2 l}=F_{i, \text { virt+ct. }}^{2 l}+\left[\frac{C_{A}}{2 \epsilon^{2}}\left(\frac{\mu_{R}^{2}}{-\hat{s}-i 0}\right)^{\epsilon}+\frac{\beta_{0}}{4 \epsilon}\right] F_{i}^{1 l}, \\
& \widetilde{F}_{i}^{3 l} \stackrel{z \rightarrow 1}{{ }^{3}} \widetilde{F}_{i, \text { virt+ct. }}^{3 l}+\left[\frac{C_{A}}{2 \epsilon^{2}}\left(\frac{\mu_{R}^{2}}{-\hat{s}-i 0}\right)^{\epsilon}+\frac{\beta_{0}}{4 \epsilon}\right] F_{i}^{2 l}+\mathcal{O}\left((1-z)^{3 / 2}\right) .
\end{aligned}
$$

The full form of the subtraction term at NNLO is known $[23,95]$ and includes a contribution proportional to $F_{i}^{1 l}$ which has been omitted here because it only affects the three-loop results beyond the considered order in the threshold expansion.

Our results for the triangle form factor at one and two loops are given in (2.8)-(2.11). At the one-loop order we determine the remaining form factors up to nrNNLO in the threshold expansion

$$
\begin{aligned}
& F_{\square}^{1 l} \stackrel{z \rightarrow 1}{\asymp}-\frac{2 \pi\left(5-8 r_{H}\right)}{3\left(1-2 r_{H}\right)^{2}}(1-z)^{3 / 2}-\frac{\pi}{15\left(1-2 r_{H}\right)^{4}}(1-z)^{5 / 2} \\
& \times\left[147-16 r_{p_{T}}-r_{H}\left(836-64 r_{p_{T}}\right)+4 r_{H}^{2}\left(409-16 r_{p_{T}}\right)-1056 r_{H}^{3}\right] \\
&+\mathcal{O}\left((1-z)^{7 / 2}\right), \\
& F_{2}^{1 l} \stackrel{z \rightarrow 1}{\asymp}-\frac{8 \pi r_{p_{T}}}{3\left(1-2 r_{H}\right)^{2}}(1-z)^{3 / 2}-\frac{4 \pi r_{p_{T}}\left(29-100 r_{H}+108 r_{H}^{2}\right)}{15\left(1-2 r_{H}\right)^{4}}(1-z)^{5 / 2} \\
&+
\end{aligned}
$$

\footnotetext{
${ }^{7}$ In spite of some notational differences, our convention is identical to [24]. There is an exact cancellation between the $\beta_{0} / \epsilon$ contribution in (C.6) and the charge and gluon field renormalization terms. This has been exploited in [24] where both effects are not written explicitly. Furthermore, the sign in the factor $(-\hat{s}-i 0)^{-\epsilon}$ has been ignored in [24] because the induced imaginary part is not relevant within the LME at the considered order in the strong coupling constant.
} 
At two-loop order the threshold expansion up to nrNNLO takes the form

$$
\begin{aligned}
& F_{\square, C_{F}}^{2 l} \stackrel{z \rightarrow 1}{\asymp}-\frac{\pi^{2}\left(5-8 r_{H}\right)}{3\left(1-2 r_{H}\right)^{2}}(1-z) \ln (1-z)+\left[\frac{\pi}{12\left(1-2 r_{H}\right)^{2}\left(1-4 r_{H}\right)}\right. \\
& \left(64-3 \pi^{2}-32 \ln (2)-\left[32+12 \pi^{2}-192 \ln (2)\right] r_{p_{T}}\right. \\
& -\left[416-12 \pi^{2}-256 \ln (2)-\left(128+48 \pi^{2}-768 \ln (2)\right) r_{p_{T}}\right] r_{H} \\
& \left.+16[41-32 \ln (2)] r_{H}^{2}-128 r_{H}^{3}\right) \\
& +\frac{8 \pi\left(1-9 r_{H}+20 r_{H}^{2}+12 r_{H}^{3}-40 r_{H}^{4}\right)}{3\left(1-2 r_{H}\right)^{2}\left(1-4 r_{H}\right)^{2}} \ln \left(2-4 r_{H}\right) \\
& +\frac{4 \pi\left(3-10 r_{H}+16 r_{H}^{2}-12 r_{H}^{3}\right)}{3\left(1-2 r_{H}\right)^{3}} \sqrt{\frac{1-r_{H}}{r_{H}}} \arctan \left(\frac{2 \sqrt{r_{H}\left(1-r_{H}\right)}}{1-2 r_{H}}\right) \\
& \left.+\frac{4 \pi\left(2-7 r_{H}+2 r_{H}^{2}\right)}{3\left(1-2 r_{H}\right)} C_{0}\left(1,4 r_{H},-1+4 r_{H} ; 0,1,1\right)\right](1-z)^{3 / 2}-\frac{4 \pi^{2}}{15\left(1-2 r_{H}\right)^{4}} \\
& \times\left(9-2 r_{p_{T}}-4 r_{H}\left(13-2 r_{p_{T}}\right)+r_{H}^{2}\left(107-8 r_{p_{T}}\right)-72 r_{H}^{3}\right)(1-z)^{2} \ln (1-z) \\
& +\mathcal{O}\left((1-z)^{5 / 2}\right) \text {, } \\
& F_{\square, C_{A}}^{2 l} \stackrel{z \rightarrow 1}{\asymp}-\frac{\pi}{6\left(1-2 r_{H}\right)^{2}}\left[2-2 \pi^{2}-4 \ln (2)+\left[2 \pi^{2}+16 \ln (2)\right] r_{H}\right. \\
& \left.+\left[8-3 \pi^{2}+24 \ln (2)\right] r_{p_{T}}\right](1-z)^{3 / 2}+\mathcal{O}\left((1-z)^{5 / 2}\right), \\
& F_{\square, C_{A}, \ln }^{2 l} \stackrel{z \rightarrow 1}{\leftrightarrows} \mathcal{O}\left((1-z)^{5 / 2}\right), \\
& F_{2, C_{F}}^{2 l} \stackrel{z \rightarrow 1}{\asymp}-\frac{4 \pi^{2} r_{p_{T}}}{3\left(1-2 r_{H}\right)^{2}}(1-z) \ln (1-z) \\
& +\left[\frac{4 \pi r_{p_{T}}}{3\left(1-2 r_{H}\right)^{2}\left(1-4 r_{H}\right)^{2}}\left(13-76 r_{H}+116 r_{H}^{2}-16 r_{H}^{3}\right)\right. \\
& -\frac{64 \pi r_{p_{T}}\left(2-15 r_{H}+37 r_{H}^{2}-36 r_{H}^{3}+16 r_{H}^{4}\right)}{3\left(1-2 r_{H}\right)^{2}\left(1-4 r_{H}\right)^{3}} \ln \left(2-4 r_{H}\right) \\
& -\frac{16 \pi r_{p_{T}} r_{H}\left(14-67 r_{H}+92 r_{H}^{2}-36 r_{H}^{3}\right)}{3\left(1-2 r_{H}\right)^{3}\left(1-4 r_{H}\right)^{2}} \sqrt{\frac{1-r_{H}}{r_{H}}} \arctan \left(\frac{2 \sqrt{r_{H}\left(1-r_{H}\right)}}{1-2 r_{H}}\right) \\
& \left.-\frac{16 \pi r_{p_{T}}\left(5-12 r_{H}+6 r_{H}^{2}+4 r_{H}^{3}\right)}{3\left(1-2 r_{H}\right)\left(1-4 r_{H}\right)^{2}} C_{0}\left(1,4 r_{H},-1+4 r_{H} ; 0,1,1\right)\right](1-z)^{3 / 2} \\
& -\frac{4 \pi^{2} r_{p_{T}}\left(7-20 r_{H}+24 r_{H}^{2}\right)}{15\left(1-2 r_{H}\right)^{4}}(1-z)^{2} \ln (1-z)+\mathcal{O}\left((1-z)^{5 / 2}\right), \\
& F_{2, C_{A}}^{2 l} \stackrel{z \rightarrow 1}{\beth^{1}}-\frac{2 \pi r_{p_{T}}}{9\left(1-2 r_{H}\right)^{2}}\left(2-3 \pi^{2}+10 \ln (2)\right)(1-z)^{3 / 2}+\mathcal{O}\left((1-z)^{5 / 2}\right), \\
& F_{2, C_{A}, \ln }^{2 l} \stackrel{z \rightarrow 1}{\varlimsup^{\prime}}-\frac{22 \pi r_{p_{T}}}{9\left(1-2 r_{H}\right)^{2}}(1-z)^{3 / 2}+\mathcal{O}\left((1-z)^{5 / 2}\right) \text {. }
\end{aligned}
$$


The three-loop form factors are determined at nrNLO in the threshold expansion

$$
\begin{aligned}
& F_{\triangle}^{3 l} \stackrel{z \rightarrow 1}{\asymp}-\frac{\pi^{3}}{6}\left(3+\pi^{2}\right) C_{F}^{2} \sqrt{1-z}+\frac{\pi^{2} C_{F}(1-z)}{8}\left\{-\beta_{0} \ln ^{2}(1-z)\right. \\
& \left.+\left[2 a_{1}+4 \beta_{0}-\left(\pi^{2}-\frac{4}{3}\right) C_{A}-\left(\frac{40}{3}-\pi^{2}\right) C_{F}\right] \ln (1-z)\right\}+\mathcal{O}\left((1-z)^{3 / 2}\right), \\
& F_{\square}^{3 l} \stackrel{z \rightarrow 1}{\asymp} \frac{\pi^{3}\left(3+\pi^{2}\right) C_{F}^{2}\left(5-8 r_{H}\right) \sqrt{1-z}}{18\left(1-2 r_{H}\right)^{2}}+\frac{\pi^{2} C_{F}(1-z)}{24\left(1-2 r_{H}\right)^{2}}\left\{\beta_{0}\left(5-8 r_{H}\right) \ln ^{2}(1-z)\right. \\
& +\left[-2\left(a_{1}+2 \beta_{0}\right)\left(5-8 r_{H}\right)-4 C_{A}\left(1-2 \ln (2)-\pi^{2}+\left(8 \ln (2)+\pi^{2}\right) r_{H}\right.\right. \\
& \left.+\left(4+12 \ln (2)-\frac{3 \pi^{2}}{2}\right) r_{p_{T}}\right)+C_{F}\left[\frac{-1}{1-4 r_{H}}+65-32 \ln (2)-3 \pi^{2}\right. \\
& -\left(32-192 \ln (2)+12 \pi^{2}\right) r_{p_{T}}-4(39-32 \ln (2)) r_{H}+32 r_{H}^{2} \\
& +\frac{32\left(1-9 r_{H}+20 r_{H}^{2}+12 r_{H}^{3}-40 r_{H}^{4}\right)}{\left(1-4 r_{H}\right)^{2}} \ln \left(2-4 r_{H}\right) \\
& +\frac{16\left(3-10 r_{H}+16 r_{H}^{2}-12 r_{H}^{3}\right)}{1-2 r_{H}} \sqrt{\frac{1-r_{H}}{r_{H}}} \arctan \left(\frac{2 \sqrt{r_{H}\left(1-r_{H}\right)}}{1-2 r_{H}}\right) \\
& \left.\left.\left.+16\left(1-2 r_{H}\right)\left(2-7 r_{H}+2 r_{H}^{2}\right) C_{0}\left(1,4 r_{H},-1+4 r_{H} ; 0,1,1\right)\right]\right] \ln (1-z)\right\} \\
& +\mathcal{O}\left((1-z)^{3 / 2}\right) \\
& F_{2}^{3 l} \stackrel{z \rightarrow 1}{\asymp} \frac{2 \pi^{3}\left(3+\pi^{2}\right) C_{F}^{2} r_{p_{T}} \sqrt{1-z}}{9\left(1-2 r_{H}\right)^{2}}+\frac{\pi^{2} C_{F} r_{p_{T}}(1-z)}{6\left(1-2 r_{H}\right)^{2}}\left\{\beta_{0} \ln ^{2}(1-z)\right. \\
& +\left[-2\left(a_{1}+2 \beta_{0}\right)-\frac{2}{3} C_{A}\left(2-3 \pi^{2}+10 \ln (2)\right)+\frac{4 C_{F}}{\left(1-4 r_{H}\right)^{2}}[13\right. \\
& -76 r_{H}+116 r_{H}^{2}-16 r_{H}^{3}-\frac{16\left(2-15 r_{H}+37 r_{H}^{2}-36 r_{H}^{3}+16 r_{H}^{4}\right)}{1-4 r_{H}} \ln \left(2-4 r_{H}\right) \\
& -\frac{4 r_{H}\left(14-67 r_{H}+92 r_{H}^{2}-36 r_{H}^{3}\right)}{1-2 r_{H}} \sqrt{\frac{1-r_{H}}{r_{H}}} \arctan \left(\frac{2 \sqrt{r_{H}\left(1-r_{H}\right)}}{1-2 r_{H}}\right) \\
& \left.\left.\left.-4\left(1-2 r_{H}\right)\left(5-12 r_{H}+6 r_{H}^{2}+4 r_{H}^{3}\right) C_{0}\left(1,4 r_{H},-1+4 r_{H} ; 0,1,1\right)\right]\right] \ln (1-z)\right\} \\
& +\mathcal{O}\left((1-z)^{3 / 2}\right), \\
& F_{2, \ln }^{3 l} \stackrel{z \rightarrow 1}{\asymp}-\frac{11 \pi^{2} C_{F} C_{A} r_{p_{T}}(1-z) \ln (1-z)}{9\left(1-2 r_{H}\right)^{2}}+\mathcal{O}\left((1-z)^{3 / 2}\right) .
\end{aligned}
$$

The logarithmic coefficients of (C.4) that are not written explicit above vanish up to and including the order $(1-z)$. The scalar triangle integral appearing above is given by

$$
C_{0}\left(1,4 r_{H},-1+4 r_{H} ; 0,1,1\right)=\int \frac{d^{d} l}{i \pi^{d / 2}} \frac{m_{t}^{2}}{\left[l^{2}\right]\left[(l+q)^{2}-m_{t}^{2}\right]\left[\left(l+q-p_{H}\right)^{2}-m_{t}^{2}\right]},
$$


with $q^{2}=m_{t}^{2}, p_{H}^{2}=m_{H}^{2}$ and $q \cdot p_{H}=m_{t}^{2}$. All boxes that appear in the hard matching computation can be reduced to at most triangles by partial fractioning since only three of the propagators in each box are linearly independent at threshold.

Open Access. This article is distributed under the terms of the Creative Commons Attribution License (CC-BY 4.0), which permits any use, distribution and reproduction in any medium, provided the original author(s) and source are credited.

\section{References}

[1] V. Ahrens, T. Becher, M. Neubert and L.L. Yang, Origin of the Large Perturbative Corrections to Higgs Production at Hadron Colliders, Phys. Rev. D 79 (2009) 033013 [arXiv:0808.3008] [INSPIRE].

[2] V. Ahrens, T. Becher, M. Neubert and L.L. Yang, Renormalization-Group Improved Prediction for Higgs Production at Hadron Colliders, Eur. Phys. J. C 62 (2009) 333 [arXiv: 0809.4283] [INSPIRE].

[3] M.A. Ebert, J.K.L. Michel and F.J. Tackmann, Resummation Improved Rapidity Spectrum for Gluon Fusion Higgs Production, JHEP 05 (2017) 088 [arXiv: 1702.00794] [INSPIRE].

[4] C. Anastasiou et al., High precision determination of the gluon fusion Higgs boson cross-section at the LHC, JHEP 05 (2016) 058 [arXiv: 1602.00695] [INSPIRE].

[5] M. Spira, A. Djouadi, D. Graudenz and P.M. Zerwas, Higgs boson production at the LHC, Nucl. Phys. B 453 (1995) 17 [hep-ph/9504378] [InSPIRE].

[6] R. Harlander and P. Kant, Higgs production and decay: Analytic results at next-to-leading order QCD, JHEP 12 (2005) 015 [hep-ph/0509189] [INSPIRE].

[7] C. Anastasiou, S. Beerli, S. Bucherer, A. Daleo and Z. Kunszt, Two-loop amplitudes and master integrals for the production of a Higgs boson via a massive quark and a scalar-quark loop, JHEP 01 (2007) 082 [hep-ph/0611236] [INSPIRE].

[8] U. Aglietti, R. Bonciani, G. Degrassi and A. Vicini, Analytic Results for Virtual QCD Corrections to Higgs Production and Decay, JHEP 01 (2007) 021 [hep-ph/0611266] [INSPIRE].

[9] S. Borowka et al., Higgs Boson Pair Production in Gluon Fusion at Next-to-Leading Order with Full Top-Quark Mass Dependence, Phys. Rev. Lett. 117 (2016) 012001 [Erratum ibid. 117 (2016) 079901] [arXiv: 1604.06447] [INSPIRE].

[10] S. Borowka et al., Full top quark mass dependence in Higgs boson pair production at NLO, JHEP 10 (2016) 107 [arXiv: 1608.04798] [INSPIRE].

[11] C. Anastasiou, C. Duhr, F. Dulat, F. Herzog and B. Mistlberger, Higgs Boson Gluon-Fusion Production in QCD at Three Loops, Phys. Rev. Lett. 114 (2015) 212001 [arXiv:1503.06056] [INSPIRE].

[12] D. de Florian and J. Mazzitelli, Higgs Boson Pair Production at Next-to-Next-to-Leading Order in QCD, Phys. Rev. Lett. 111 (2013) 201801 [arXiv:1309.6594] [INSPIRE].

[13] J. Grigo, K. Melnikov and M. Steinhauser, Virtual corrections to Higgs boson pair production in the large top quark mass limit, Nucl. Phys. B 888 (2014) 17 [arXiv:1408.2422] [INSPIRE]. 
[14] R. Boughezal, F. Caola, K. Melnikov, F. Petriello and M. Schulze, Higgs boson production in association with a jet at next-to-next-to-leading order in perturbative QCD, JHEP 06 (2013) 072 [arXiv: 1302.6216] [INSPIRE].

[15] X. Chen, T. Gehrmann, E.W.N. Glover and M. Jaquier, Precise QCD predictions for the production of Higgs + jet final states, Phys. Lett. B 740 (2015) 147 [arXiv:1408.5325] [INSPIRE].

[16] R. Boughezal, F. Caola, K. Melnikov, F. Petriello and M. Schulze, Higgs boson production in association with a jet at next-to-next-to-leading order, Phys. Rev. Lett. 115 (2015) 082003 [arXiv: 1504.07922] [INSPIRE].

[17] R. Boughezal, C. Focke, W. Giele, X. Liu and F. Petriello, Higgs boson production in association with a jet at NNLO using jettiness subtraction, Phys. Lett. B 748 (2015) 5 [arXiv: 1505.03893] [INSPIRE].

[18] R.V. Harlander and K.J. Ozeren, Top mass effects in Higgs production at next-to-next-to-leading order QCD: Virtual corrections, Phys. Lett. B 679 (2009) 467 [arXiv: 0907.2997] [INSPIRE].

[19] A. Pak, M. Rogal and M. Steinhauser, Virtual three-loop corrections to Higgs boson production in gluon fusion for finite top quark mass, Phys. Lett. B 679 (2009) 473 [arXiv: 0907.2998] [INSPIRE].

[20] R.V. Harlander and K.J. Ozeren, Finite top mass effects for hadronic Higgs production at next-to-next-to-leading order, JHEP 11 (2009) 088 [arXiv:0909.3420] [INSPIRE].

[21] A. Pak, M. Rogal and M. Steinhauser, Finite top quark mass effects in NNLO Higgs boson production at LHC, JHEP 02 (2010) 025 [arXiv:0911.4662] [INSPIRE].

[22] R.V. Harlander, H. Mantler, S. Marzani and K.J. Ozeren, Higgs production in gluon fusion at next-to-next-to-leading order QCD for finite top mass, Eur. Phys. J. C 66 (2010) 359 [arXiv: 0912.2104] [INSPIRE].

[23] J. Grigo, J. Hoff and M. Steinhauser, Higgs boson pair production: top quark mass effects at NLO and NNLO, Nucl. Phys. B 900 (2015) 412 [arXiv: 1508.00909] [INSPIRE].

[24] G. Degrassi, P.P. Giardino and R. Gröber, On the two-loop virtual QCD corrections to Higgs boson pair production in the Standard Model, Eur. Phys. J. C 76 (2016) 411 [arXiv: 1603.00385] [INSPIRE].

[25] A. Hasselhuhn, T. Luthe and M. Steinhauser, On top quark mass effects to $g g \rightarrow Z H$ at NLO, JHEP 01 (2017) 073 [arXiv: 1611.05881] [INSPIRE].

[26] J.M. Campbell, R.K. Ellis, M. Czakon and S. Kirchner, Two loop correction to interference in $g g \rightarrow Z Z$, JHEP 08 (2016) 011 [arXiv: 1605.01380] [INSPIRE].

[27] F. Caola, M. Dowling, K. Melnikov, R. Röntsch and L. Tancredi, QCD corrections to vector boson pair production in gluon fusion including interference effects with off-shell Higgs at the LHC, JHEP 07 (2016) 087 [arXiv: 1605. 04610] [INSPIRE].

[28] J. Fleischer and O.V. Tarasov, Calculation of Feynman diagrams from their small momentum expansion, Z. Phys. C 64 (1994) 413 [hep-ph/9403230] [INSPIRE].

[29] D.J. Broadhurst, J. Fleischer and O.V. Tarasov, Two loop two point functions with masses: Asymptotic expansions and Taylor series, in any dimension, Z. Phys. C 60 (1993) 287 [hep-ph/9304303] [INSPIRE]. 
[30] K.G. Chetyrkin, R. Harlander and M. Steinhauser, Singlet polarization functions at $\mathcal{O}\left(\alpha_{s}^{2}\right)$, Phys. Rev. D 58 (1998) 014012 [hep-ph/9801432] [INSPIRE].

[31] Y. Kiyo, A. Maier, P. Maierhöfer and P. Marquard, Reconstruction of heavy quark current correlators at $O\left(\alpha_{s}^{3}\right)$, Nucl. Phys. B 823 (2009) 269 [arXiv:0907.2120] [inSPIRE].

[32] A.H. Hoang, V. Mateu and S. Mohammad Zebarjad, Heavy Quark Vacuum Polarization Function at $O\left(\alpha_{s}^{2}\right)$ and $O\left(\alpha_{s}^{3}\right)$, Nucl. Phys. B 813 (2009) 349 [arXiv:0807.4173] [InSPIRE].

[33] A. Maier and P. Marquard, Life of П, 2017, arXiv:1710.03724, [INSPIRE].

[34] A. Djouadi, W. Kilian, M. Mühlleitner and P.M. Zerwas, Production of neutral Higgs boson pairs at LHC, Eur. Phys. J. C 10 (1999) 45 [hep-ph/9904287] [INSPIRE].

[35] M.J. Dolan, C. Englert and M. Spannowsky, Higgs self-coupling measurements at the LHC, JHEP 10 (2012) 112 [arXiv:1206.5001] [INSPIRE].

[36] J. Baglio, A. Djouadi, R. Gröber, M.M. Mühlleitner, J. Quevillon and M. Spira, The measurement of the Higgs self-coupling at the LHC: theoretical status, JHEP 04 (2013) 151 [arXiv: 1212.5581] [INSPIRE].

[37] ATLAS, CMS collaborations, Measurements of the Higgs boson production and decay rates and constraints on its couplings from a combined ATLAS and CMS analysis of the LHC pp collision data at $\sqrt{s}=7$ and $8 \mathrm{TeV}$, JHEP 08 (2016) 045 [arXiv:1606.02266] [INSPIRE].

[38] ATLAS collaboration, Evidence for the $H \rightarrow b \bar{b}$ decay with the ATLAS detector, ATLAS-CONF-2017-041.

[39] CMS collaboration, Evidence for the Higgs boson decay to a bottom quark-antiquark pair, arXiv: 1709.07497 [INSPIRE].

[40] ATLAS collaboration, Projected sensitivity to non-resonant Higgs boson pair production in the $b \bar{b} b \bar{b}$ final state using proton-proton collisions at HL-LHC with the ATLAS detector, ATL-PHYS-PUB-2016-024.

[41] CMS collaboration, Search for Higgs boson pair production in the final state containing two photons and two bottom quarks in proton-proton collisions at $\sqrt{s}=13 \mathrm{TeV}$, CMS-PAS-HIG-17-008.

[42] M. McCullough, An Indirect Model-Dependent Probe of the Higgs Self-Coupling, Phys. Rev. D 90 (2014) 015001 [arXiv:1312.3322] [INSPIRE].

[43] M. Gorbahn and U. Haisch, Indirect probes of the trilinear Higgs coupling: $g g \rightarrow h$ and $h \rightarrow \gamma \gamma$, JHEP 10 (2016) 094 [arXiv: 1607.03773] [INSPIRE].

[44] G. Degrassi, P.P. Giardino, F. Maltoni and D. Pagani, Probing the Higgs self coupling via single Higgs production at the LHC, JHEP 12 (2016) 080 [arXiv:1607.04251] [INSPIRE].

[45] W. Bizon, M. Gorbahn, U. Haisch and G. Zanderighi, Constraints on the trilinear Higgs coupling from vector boson fusion and associated Higgs production at the LHC, JHEP 07 (2017) 083 [arXiv: 1610.05771] [INSPIRE].

[46] G. Degrassi, M. Fedele and P.P. Giardino, Constraints on the trilinear Higgs self coupling from precision observables, JHEP 04 (2017) 155 [arXiv: 1702.01737] [INSPIRE].

[47] G.D. Kribs, A. Maier, H. Rzehak, M. Spannowsky and P. Waite, Electroweak oblique parameters as a probe of the trilinear Higgs boson self-interaction, Phys. Rev. D 95 (2017) 093004 [arXiv: 1702.07678] [INSPIRE]. 
[48] L. Di Luzio, R. Gröber and M. Spannowsky, Maxi-sizing the trilinear Higgs self-coupling: how large could it be?, Eur. Phys. J. C 77 (2017) 788 [arXiv:1704.02311] [InSPIRE].

[49] K. Melnikov, L. Tancredi and C. Wever, Two-loop gg $\rightarrow$ Hg amplitude mediated by a nearly massless quark, JHEP 11 (2016) 104 [arXiv:1610.03747] [INSPIRE].

[50] K. Melnikov, L. Tancredi and C. Wever, Two-loop amplitudes for $q g \rightarrow H q$ and $q \bar{q} \rightarrow H g$ mediated by a nearly massless quark, Phys. Rev. D 95 (2017) 054012 [arXiv:1702.00426] [INSPIRE].

[51] M. Beneke, A.P. Chapovsky, A. Signer and G. Zanderighi, Effective theory approach to unstable particle production, Phys. Rev. Lett. 93 (2004) 011602 [hep-ph/0312331] [InSPIRE].

[52] M. Beneke, A.P. Chapovsky, A. Signer and G. Zanderighi, Effective theory calculation of resonant high-energy scattering, Nucl. Phys. B 686 (2004) 205 [hep-ph/0401002] [INSPIRE].

[53] M. Beneke, P. Falgari and C. Schwinn, Soft radiation in heavy-particle pair production: All-order colour structure and two-loop anomalous dimension, Nucl. Phys. B $\mathbf{8 2 8}$ (2010) 69 [arXiv: 0907.1443] [INSPIRE].

[54] M. Beneke, P. Falgari and C. Schwinn, Threshold resummation for pair production of coloured heavy (s)particles at hadron colliders, Nucl. Phys. B 842 (2011) 414 [arXiv: 1007.5414] [INSPIRE].

[55] P. Falgari, C. Schwinn and C. Wever, NLL soft and Coulomb resummation for squark and gluino production at the LHC, JHEP 06 (2012) 052 [arXiv:1202.2260] [INSPIRE].

[56] M. Beneke, P. Falgari, C. Schwinn, A. Signer and G. Zanderighi, Four-fermion production near the W pair production threshold, Nucl. Phys. B 792 (2008) 89 [arXiv:0707.0773] [INSPIRE].

[57] S. Actis, M. Beneke, P. Falgari and C. Schwinn, Dominant NNLO corrections to four-fermion production near the W-pair production threshold, Nucl. Phys. B 807 (2009) 1 [arXiv:0807.0102] [INSPIRE].

[58] M. Beneke, B. Jantzen and P. Ruiz-Femenía, Electroweak non-resonant NLO corrections to $e^{+} e^{-} \rightarrow W^{+} W^{-} b \bar{b}$ in the $t \bar{t}$ resonance region, Nucl. Phys. B 840 (2010) 186 [arXiv: 1004.2188] [INSPIRE].

[59] B. Jantzen and P. Ruiz-Femenía, Next-to-next-to-leading order nonresonant corrections to threshold top-pair production from $e^{+} e^{-}$collisions: Endpoint-singular terms, Phys. Rev. D 88 (2013) 054011 [arXiv: 1307.4337] [INSPIRE].

[60] M. Beneke, A. Maier, T. Rauh and P. Ruiz-Femenía, Non-resonant and electroweak NNLO correction to the $e^{+} e^{-}$top anti-top threshold, JHEP 02 (2018) 125 [arXiv:1711.10429] [INSPIRE].

[61] M. Beneke, P. Falgari, S. Klein and C. Schwinn, Hadronic top-quark pair production with NNLL threshold resummation, Nucl. Phys. B 855 (2012) 695 [arXiv:1109.1536] [INSPIRE].

[62] M. Beneke et al., Inclusive Top-Pair Production Phenomenology with TOPIXS, JHEP 07 (2012) 194 [arXiv:1206.2454] [INSPIRE].

[63] M. Beneke, J. Piclum, C. Schwinn and C. Wever, NNLL soft and Coulomb resummation for squark and gluino production at the LHC, JHEP 10 (2016) 054 [arXiv:1607.07574] [INSPIRE]. 
[64] A. Pineda and J. Soto, Effective field theory for ultrasoft momenta in NRQCD and NRQED, Nucl. Phys. Proc. Suppl. 64 (1998) 428 [hep-ph/9707481] [INSPIRE].

[65] A. Pineda and J. Soto, The Lamb shift in dimensional regularization, Phys. Lett. B 420 (1998) 391 [hep-ph/9711292] [INSPIRE].

[66] M. Beneke, New results on heavy quarks near threshold, in Continuous advances in QCD. Proceedings, 3rd Workshop, QCD'98, Minneapolis, U.S.A., April 16-19, 1998, pp. 293-309, hep-ph/9806429, [INSPIRE].

[67] M. Beneke, A. Signer and V.A. Smirnov, Top quark production near threshold and the top quark mass, Phys. Lett. B 454 (1999) 137 [hep-ph/9903260] [INSPIRE].

[68] N. Brambilla, A. Pineda, J. Soto and A. Vairo, Potential NRQCD: An Effective theory for heavy quarkonium, Nucl. Phys. B 566 (2000) 275 [hep-ph/9907240] [INSPIRE].

[69] M. Beneke, Y. Kiyo and K. Schuller, Third-order correction to top-quark pair production near threshold I. Effective theory set-up and matching coefficients, arXiv:1312.4791 [INSPIRE].

[70] C.W. Bauer, S. Fleming and M.E. Luke, Summing Sudakov logarithms in $B \rightarrow X_{s} \gamma$ in effective field theory, Phys. Rev. D 63 (2000) 014006 [hep-ph/0005275] [InSPIRE].

[71] C.W. Bauer, S. Fleming, D. Pirjol and I.W. Stewart, An Effective field theory for collinear and soft gluons: Heavy to light decays, Phys. Rev. D 63 (2001) 114020 [hep-ph/0011336] [INSPIRE].

[72] C.W. Bauer, D. Pirjol and I.W. Stewart, Soft collinear factorization in effective field theory, Phys. Rev. D 65 (2002) 054022 [hep-ph/0109045] [INSPIRE].

[73] M. Beneke, A.P. Chapovsky, M. Diehl and T. Feldmann, Soft collinear effective theory and heavy to light currents beyond leading power, Nucl. Phys. B 643 (2002) 431 [hep-ph/0206152] [INSPIRE].

[74] M. Beneke and T. Feldmann, Multipole expanded soft collinear effective theory with nonAbelian gauge symmetry, Phys. Lett. B 553 (2003) 267 [hep-ph/0211358] [INSPIRE].

[75] T. Becher, A. Broggio and A. Ferroglia, Introduction to Soft-Collinear Effective Theory, Lect. Notes Phys. 896 (2015) pp.1 [arXiv:1410.1892] [INSPIRE].

[76] M. Beneke, J. Piclum and T. Rauh, P-wave contribution to third-order top-quark pair production near threshold, Nucl. Phys. B 880 (2014) 414 [arXiv:1312.4792] [INSPIRE].

[77] M. Beneke, Y. Kiyo, P. Marquard, A. Penin, J. Piclum and M. Steinhauser, Next-to-Next-to-Next-to-Leading Order QCD Prediction for the Top Antitop S-Wave Pair Production Cross section Near Threshold in $e^{+} e^{-}$Annihilation, Phys. Rev. Lett. 115 (2015) 192001 [arXiv: 1506.06864] [INSPIRE].

[78] O.J.P. Eboli, G.C. Marques, S.F. Novaes and A.A. Natale, Twin Higgs Boson Production, Phys. Lett. B 197 (1987) 269 [INSPIRE].

[79] E.W.N. Glover and J.J. van der Bij, Higgs Boson Pair Production Via Gluon Fusion, Nucl. Phys. B 309 (1988) 282 [inSPIRE].

[80] T. Plehn, M. Spira and P.M. Zerwas, Pair production of neutral Higgs particles in gluon-gluon collisions, Nucl. Phys. B 479 (1996) 46 [Erratum ibid. B 531 (1998) 655] [hep-ph/9603205] [INSPIRE].

[81] S. Dawson, S. Dittmaier and M. Spira, Neutral Higgs boson pair production at hadron colliders: QCD corrections, Phys. Rev. D 58 (1998) 115012 [hep-ph/9805244] [INSPIRE]. 
[82] F. Maltoni, E. Vryonidou and M. Zaro, Top-quark mass effects in double and triple Higgs production in gluon-gluon fusion at NLO, JHEP 11 (2014) 079 [arXiv:1408.6542] [INSPIRE].

[83] R. Frederix et al., Higgs pair production at the LHC with NLO and parton-shower effects, Phys. Lett. B 732 (2014) 142 [arXiv:1401.7340] [InSPIRE].

[84] NNPDF collaboration, R.D. Ball et al., Parton distributions for the LHC Run II, JHEP 04 (2015) 040 [arXiv: 1410.8849] [INSPIRE].

[85] D.Y. Shao, C.S. Li, H.T. Li and J. Wang, Threshold resummation effects in Higgs boson pair production at the LHC, JHEP 07 (2013) 169 [arXiv:1301.1245] [INSPIRE].

[86] D. de Florian and J. Mazzitelli, Higgs pair production at next-to-next-to-leading logarithmic accuracy at the LHC, JHEP 09 (2015) 053 [arXiv: 1505.07122] [INSPIRE].

[87] J.J. Moré, B.S. Garbow and K.E. Hillstrom, User Guide for MINPACK-1, Argonne National Laboratory Report ANL-80-74, Argonne, Ill. (1980).

[88] J. Moré, D. Sorenson, B. Garbow and K. Hillstrom, The MINPACK Project, Sources and Development of Mathematical Software, W. Cowell ed., Prentice-Hall (1984) [ISBN: 0-13-823501-5].

[89] Michael Spira's webpage, http://tiger.web.psi.ch.

[90] G. Heinrich, S.P. Jones, M. Kerner, G. Luisoni and E. Vryonidou, NLO predictions for Higgs boson pair production with full top quark mass dependence matched to parton showers, JHEP 08 (2017) 088 [arXiv: 1703.09252] [INSPIRE].

[91] R. Gröber, M. Mühlleitner, M. Spira and J. Streicher, NLO QCD Corrections to Higgs Pair Production including Dimension-6 Operators, JHEP 09 (2015) 092 [arXiv:1504.06577] [INSPIRE].

[92] R. Mueller and D.G. Öztürk, On the computation of finite bottom-quark mass effects in Higgs boson production, JHEP 08 (2016) 055 [arXiv: 1512.08570] [INSPIRE].

[93] D. de Florian and J. Mazzitelli, A next-to-next-to-leading order calculation of soft-virtual cross sections, JHEP 12 (2012) 088 [arXiv:1209.0673] [inSPIRE].

[94] A.O.G. Källén and A. Sabry, Fourth order vacuum polarization, Kong. Dan. Vid. Sel. Mat. Fys. Med. 29 (1955) 1 [INSPIRE].

[95] S. Catani, The singular behavior of QCD amplitudes at two loop order, Phys. Lett. B 427 (1998) 161 [hep-ph/9802439] [INSPIRE]. 